JOURNAL OF

SYMPLECTIC GEOMETRY

Volume 1, Number 1, 121-169, 2001

\title{
Grothendieck Groups of Poisson Vector Bundles
}

\author{
VIKTOR L. GinZBURG ${ }^{1}$
}

\begin{abstract}
A new invariant of Poisson manifolds, a Poisson $K$-ring, is introduced. Evidence is given that this invariant is more tractable than such invariants as Poisson (co)homology. A version of this invariant is also defined for arbitrary Lie algebroids. Basic properties of the Poisson $K$-ring are proved and the Poisson $K$-rings are calculated for a number of examples. In particular, for the zero Poisson structure the $K$-ring is the ordinary $K^{0}$-ring of the manifold and for the dual space to a Lie algebra the $K$-ring is the ring of virtual representations of the Lie algebra. It is also shown that the $K$-ring is an invariant of Morita equivalence. Moreover, the $K$-ring is a functor on a category, the weak Morita category, which generalizes the notion of Morita equivalence of Poisson manifolds.
\end{abstract}

\section{Introduction.}

Poisson manifolds have a wide variety of invariants, almost all of which, with few notable exceptions, are extremely hard to compute. For instance, Poisson (co)homology and its various "derivatives" are not calculated explicitly even for dual spaces to semisimple Lie algebras of non-compact type. (In fact, it is not even clear in what terms the result should be expressed.) The goal of this paper is to introduce a more tractable invariant of Poisson manifolds, a Poisson $K$-ring. Alternatively, this paper is devoted to the study of representations of the algebroid of differential forms on a Poisson manifold, albeit the study is oriented toward a very specific and narrow objective. This work fits in the subject of Poisson topology which we loosely define by simply listing some of the relevant publications: [Be, Cr1, Fe1, ELW, GG, GL, We1, We2, Xu1].

\footnotetext{
${ }^{1}$ The work was partially supported by the NSF and by the faculty research funds of the UC Santa Cruz.
} 


\subsection{Poisson $K$-theory.}

Poisson geometry occupies an intermediate place between geometry of smooth manifolds and non-commutative geometry. The $K$-theory, the most robust and algebraic of all geometric "cohomology theories", is fairly easy to define in either smooth and non-commutative settings, and it should also have an analogue in the context of Poisson geometry.

Leaving for a moment the question of its construction aside, we note that it is reasonable to expect a "correct" Poisson $K^{0}$-functor $K_{\pi}$ to satisfy the following three conditions:

(K1) When $P$ is a Poisson manifold with zero Poisson structure, the Poisson $K$-ring of $P$ is equal to the ordinary topological $K$-ring of $P$, i.e., $K_{\pi}(P)=K(P)$. Moreover, $K_{\pi}(P)=K(B)$, if $P=B \times F$ with $\pi=0 \oplus \omega^{-1}$, where $B$ is a manifold with zero Poisson structure and $(F, \omega)$ is a simply connected symplectic manifold. ${ }^{2}$

(K2) When $P$ is symplectic with a sufficiently nice (e.g., finite) fundamental group, $K_{\pi}(P)=R\left(\pi_{1}(P)\right)$, where $R$ denotes the ring of representations. In general, in the symplectic case, $K_{\pi}(P)$ should lie in the range between $R\left(\pi_{1}(P)\right)$ and the Grothendieck ring generated by the semiring of path-connected components of $\operatorname{Rep}\left(\pi_{1}(P)\right.$ ). (Here Rep stands for the space of representations.)

(K3) Let $\mathfrak{g}$ be a Lie algebra. The ring $K_{\pi}\left(\mathfrak{g}^{*}\right)$ should reflect representation properties of $\mathfrak{g}$ and one should have $K_{\pi}\left(\mathfrak{g}^{*}\right)=R(\mathfrak{g})$, when $\mathfrak{g}$ is sufficiently nice, e.g., semisimple. In general, $K_{\pi}\left(\mathfrak{g}^{*}\right)$ should lie in the range between $R(\mathfrak{g})$ and the Grothendieck ring generated by the path-connected components of $\operatorname{Rep}(\mathfrak{g})$.

These three requirements deserve some discussion.

Note that the requirement (K1), which we accept in this paper unconditionally, is incompatible with the requirement that $K_{\pi}\left(\mathfrak{g}^{*}\right)=R(\mathfrak{g})$ for all $\mathfrak{g}$. Indeed, for $\mathfrak{g}=\mathbb{R}$, we have $K_{\pi}(\mathbb{R})=\mathbb{Z}$ by $(\mathrm{K} 1)$, whereas $R(\mathbb{R})$ is a free group generated by $\mathbb{R}$. This simple observation is indicative of the difficulties arising in the definition of a Poisson $K$-ring and is the main reason that the third condition is stated somewhat vaguely.

\footnotetext{
${ }^{2}$ Here we adopt the standard topological convention that a simply connected space is by definition connected. We also consider only the $K^{0}$-functor: $K(B)=$ $K^{0}(B)$ and $K(P)=K^{0}(P)$.
} 
Furthermore, we emphasize that in (K1) we do not require that $K_{\pi}(P)=$ $K(B)$, whenever the symplectic foliation of $P$ is a fibration over $B$ with simply connected fibers. In fact, Poisson $K$-rings defined in this paper are sufficiently sensitive to detect whether or not the Poisson structure on $P$ varies from fiber to fiber.

The similarity between the requirements (K2) and (K3) arises from one of the main principles of Poisson geometry. In this connection we simply quote Alan Weinstein, [We3]: It is tempting to think of the symplectic manifold $N$ as the "dual of the Lie algebra of $\pi_{1}(N)$ ". Conversely, a simply connected group with Lie algebra $\mathfrak{g}$ can be viewed as the "Poisson fundamental group of $\mathfrak{g}^{*}$. In fact, $K$-rings considered in this paper should be thought of as "the rings of virtual representations of the Lie algebra whose dual is $P$ ".

A Poisson structure gives rise to a (singular) foliation on the manifold. A $K$-theory of foliated manifolds is defined in the context of non-commutative geometry, [Con1, Con2]. This suggests that a "correct" Poisson $K$-ring should also be constructed using non-commutative geometry techniques. However, this idea encounters a number of technical problems.

Here we take a more naive approach, introduce Poisson vector bundles, and define two versions of a Poisson $K$-ring as the Grothendieck rings for suitable classes of vector bundles and their equivalences. As a consequence, the resulting $K$-rings cannot be expected to give meaningful results for Poisson manifolds with severely non-Hausdorff spaces of symplectic leaves. On the other hand, for manifolds whose spaces of leaves are only mildly nonHausdorff, these rings appear to be "correct". (This is the case, for example, for $\mathfrak{g}^{*}$, when $\mathfrak{g}$ is semisimple.) In brief, the $K$-rings introduced in this paper are related to the "correct" one, which may fail to exist, in the same way as the classical $K$-theory is related to the non-commutative one.

The objects we refer to as Poisson vector bundles are well known in a different context as representations of Lie algebroids. Already in the simplest examples, they form too large a semi-ring to produce a Grothendieck ring satisfying (K1)-(K3). We deal with this problem in two ways: by imposing an extra condition on representations and by relaxing the equivalence relation. The resulting semi-rings are then suitable for our purposes.

\subsection{Organization of the paper; conventions.}

In Section 2 we define Poisson vector bundles and $K$-rings, prove their basic properties, and examine a number of examples. No reference to algebroids and groupoids and their representations is made here. In Section 3, we put the constructions of Section 2 in the context of representations of algebroids 
and groupoids. We also recall the definition of secondary characteristic classes due to Fernandes, [Fe1, Fe2], and Crainic, [Cr1], and establish their properties important for what follows. Section 4 is entirely devoted to the calculation of the Poisson $K$-rings for the dual space to a Lie algebra. In Section 5 we prove Morita invariance of Poisson $K$-rings. In Sections 6 and 7 we partially generalize this result to representations of algebroids and introduce a new category (the weak Morita category) on which algebroid cohomology and one of our $K$-rings are functors.

A few meaningful questions and calculations are entirely left out in the present account. Among these are the calculations of $K$-rings for BruhatPoisson structures and for irrational affine Poisson structures on tori. In fact, getting explicit answers in these examples would be the ultimate validity test for $K$-rings as tractable invariants of Poisson manifolds. Neither do we touch the problem of finding an analogue of the Bott periodicity or defining $K^{n}$-functors. We only consider Poisson and algebroid versions of $K^{0}$. Note also that our $K$-rings are defined using a very narrow class of vector bundles over a Poisson manifolds or representations of an algebroid. There are some indications that more general objects, representations up to homotopy, are at least as natural in the context of Poisson or algebroid geometry as genuine representations; see [Cr2, ELW, Fe1, Fe2]. We do not work with this bigger class, for the resulting Grothendieck rings appear to be more difficult to calculate even in simple examples.

Much of what follows holds simultaneously for real or orthogonal or complex or unitary vector bundles with obvious modifications. Hence, we only specify the class of vector bundles when the distinction is relevant. The reader may view the case of complex vector bundles as the "default setting" throughout this paper. All algebroids and groupoids are assumed to be smooth, i.e., Lie algebroids and groupoids.

Acknowledgments. The author is grateful to Rui Loja Fernandes, Steve Ferry, Leonid Polterovich, and Alan Weinstein for useful discussions. He

would also like to express his gratitude to the EPFL for its hospitality during the period when some parts of this paper were written.

\section{Poisson Vector Bundles.}

\subsection{Definitions.}

Let $P$ be a Poisson manifold with Poisson structure $\pi$. 
Definition 2.1. A Poisson vector bundle $E \rightarrow P$ over $P$ is a vector bundle together with a bracket $\{\}:, C^{\infty}(P) \times \Gamma(E) \rightarrow \Gamma(E)$ which turns $\Gamma(E)$ into a Lie algebra module over $C^{\infty}(P)$ and such that the following two Leibniz identities hold

$$
\begin{aligned}
& \{f, h s\}=\{f, h\} s+h\{f, s\} \text { and } \\
& \{f h, s\}=f\{h, s\}+h\{f, s\}
\end{aligned}
$$

for all $f$ and $h$ in $C^{\infty}(P)$ and all $s \in \Gamma(E)$.

Isomorphisms of Poisson vector bundles are defined as isomorphisms of ordinary vector bundles preserving the Poisson vector bundle structure. We denote the semi-ring of isomorphism classes of Poisson vector bundles over $P$ by $\operatorname{Vect}_{\pi}(P)$.

The restriction of a Poisson vector bundle to a symplectic leaf does not have, in general, a natural structure of a Poisson vector bundle. For example, consider the zero Poisson structure on $P$. The fiber $E_{p}$ over $p \in P$ is the restriction of $E$ to the leaf $p$. The action of $f \in C^{\infty}(P)$ on $E_{p}$ depends in general on the differential $d f$ at $p$ and is not determined by $f(p)$. (In fact, this action is independent of $f(p)$.) This shows that $E_{p}$ does not naturally inherit the Poisson vector bundle structure from $E$. More generally, the pull-back of a Poisson vector bundle under a Poisson map does not naturally receive the structure of a Poisson vector bundle, i.e., Vect ${ }_{\pi}$ is not a functor for Poisson maps.

The two Leibniz identities in the definition of Poisson vector bundles play very different roles. The identity (L1) appears to be absolutely necessary and can be thought of as the definition of a Poisson module over $C^{\infty}(P)$. The second identity (L2) is more technical as the following obvious observation indicates:

Proposition 2.2. The second Leibniz identity (L2) is equivalent to requiring that $\{f, s\}(p)$, for all points $p \in P$, depends only on the differential $d f(p)$ (and, by (L1), on the 1-jet of $s$ at $p$ ). The second Leibniz identity is also equivalent to the following two conditions:

$$
\{1, s\}=0 \quad \text { for all } \quad s \in \Gamma(E)
$$

and

$$
\{f, s\}(p)=0 \quad \text { for all } \quad s \in \Gamma(E),
$$

whenever $f$ vanishes at $p$ up to second order. 
Example 2.3. Let $E$ be the trivial vector bundle $P \times \mathbb{C}^{k}$ and let us identify $\Gamma(E)$ with $C^{\infty}\left(P, \mathbb{C}^{k}\right)$. By setting the bracket $\{\}:, C^{\infty}(P) \times \Gamma(E) \rightarrow \Gamma(E)$ to be the component-wise Poisson bracket on $P$, we turn $E$ into a Poisson vector bundle, which we refer to as Poisson-trivial. A Poisson vector bundle $E$ is said to be locally Poisson-trivial if $E$ is locally isomorphic to a Poissontrivial vector bundle. Poisson vector bundles are rarely locally Poissontrivial (see examples below).

Remark 2.4. A complex Poisson vector bundle $E$ is said to be Hermitian or unitary if $E$ is equipped with a Hermitian inner product $\langle$,$\rangle such that$

$$
\left\{f,\left\langle s_{1}, s_{2}\right\rangle\right\}=\left\langle\left\{f, s_{1}\right\}, s_{2}\right\rangle+\left\langle s_{1},\left\{\bar{f}, s_{2}\right\}\right\rangle
$$

for all $f \in C^{\infty}(P)$ and all sections $s_{1}$ and $s_{2}$ of $E$. In contrast with ordinary vector bundles, not every Poisson vector bundle can be made Hermitian; see Examples 2.6, 2.7, and 2.19. The definition of Euclidean Poisson vector bundles is similar.

We emphasize that unless stated otherwise Vect $_{\pi}$ means either one out of four semi-rings: complex, real, Hermitian, or Euclidean Poisson vector bundles. Of course, in every formula or equality vector bundles or representations are assumed to be in the same class.

Let us illustrate these definitions by a series of examples.

\subsection{Basic examples.}

Example 2.5. Assume that the Poisson structure on $P$ is zero. Then every vector bundle over $P$ with trivial bracket is a Poisson vector bundle.

Example 2.6. Let $E$ be a bundle with a flat connection $\nabla$ over a Poisson manifold $P$. Then $E$ is a Poisson bundle over $P$ with $\{f, s\}=\nabla_{X_{f}} s$. This Poisson vector bundle is Hermitian if and only if $\nabla$ is a Hermitian connection.

Example 2.7. Let $\mathfrak{g}$ be a finite-dimensional Lie algebra and let $P=\mathfrak{g}^{*}$ with the standard Poisson structure. Let $V$ be a finite-dimensional representation of $\mathfrak{g}$. Set $E=\mathfrak{g}^{*} \times V$ and $\{f, s\}(p)=d f(p) \cdot s(p)+\left(L_{X_{f}} s\right)(p)$. Here the first term denotes the action of $d f(p) \in T_{p}^{*} \mathfrak{g}^{*}=\mathfrak{g}$ on $s(p) \in V$ and the second term is just the Lie derivative of the vector valued function $s$. It is easy to see that $E$ is a Poisson vector bundle over $\mathfrak{g}^{*}$. This bundle is (locally) Poisson-trivial if and only if $V$ is a trivial representation; $E$ is Hermitian if and only $V$ is a unitary representation. 
The next two examples are more interesting from the Poisson geometry perspective.

Example 2.8. Let $G$ be a Poisson Lie group and let $G^{*}$ be its dual, which we assume to be simply connected. Then every representation of $G$ gives rise to a Poisson vector bundle over $G^{*}$ similarly to Example 2.7. For instance, when $G$ is compact semisimple, $G^{*}$ is isomorphic to $\mathfrak{g}^{*}$, [Al, GW], and Example 2.7 applies. For an arbitrary $G$ we give an explicit construction of this Poisson vector bundle in Example 2.15.

Example 2.9. Let $K$ be a compact Poisson Lie group with the standard Poisson structure $\pi$ and let $T$ be the maximal torus in $K$ such that $\pi$ vanishes along $T$. The Poisson structure $\pi$ descends to the quotient space $P=K / T$ making it into a Poisson manifold; see [LW]. Let $V$ be a representation of $T$. The vector bundle $E=K \times_{T} V$ over $P$ is Poisson with the bracket defined as follows. Let us identify $\Gamma(E)$ with the space of $T$-equivariant functions $K \rightarrow V$ and $C^{\infty}(P)$ with the space of $T$-invariant functions $K \rightarrow \mathbb{C}$. Then $\{f, s\}$ is just the bracket of $f$ and $s$ thought of as functions on $K$, i.e., $L_{X_{f}} s$, where $X_{f}$ is the Hamiltonian vector field of $f$ on $K$. (It is not hard to see that $\{f, s\}$ so-defined is again $T$-equivariant and hence a section of $E$.)

The next two examples illustrate what happens when the requirement (L2) is omitted. (See also Example 3.7 below.)

Example 2.10. Let $E=T P$ and $\{f, s\}=\left[X_{f}, s\right]$, where $X_{f}$ is the Hamiltonian vector field of $f$. This is not a Poisson vector bundle unless $\pi=0$. The bracket satisfies (L1), but not (L2): the condition (2) fails if $\pi \neq 0$.

Example 2.11. Let $P$ be symplectic with integral symplectic form $\omega$. Let $\mathbb{L} \rightarrow P$ be a pre-quantum complex line bundle over $P$, i.e., a line bundle with $c_{1}(\mathbb{L})=[\omega]$ and let $\nabla$ be a Hermitian connection on $\mathbb{L}$ with curvature $-\omega$. Set $\{f, s\}=-\nabla_{X_{f}} s+\sqrt{-1} f s$. Then, $\mathbb{L}$ is not a Poisson vector bundle. The bracket satisfies (L1) but not (L2): the condition (1) fails. The construction of pre-quantization generalizes to Poisson algebras associated with closed two-forms or Dirac structures (see [GGK, Chapter 6]) and to some Poisson manifolds, [Hu, Va]. However, Poisson manifolds which are pre-quantizable in this sense are rather rare: the line bundle $\mathbb{L}$ over $(P, \pi)$ exists if and only if $\pi$ is the image under $\pi^{\#}$ of an integral closed two-form on $P,[\mathrm{Hu}, \mathrm{Va}]$. For example, the dual space of the Lie algebra of a compact semisimple Lie group is never pre-quantizable. 
Remark 2.12. Example 2.11 shows that the class of vector bundles over a Poisson manifold satisfying less restrictive conditions than (L1) and (L2) may also be of interest. For instance, to include pre-quantization of Poisson manifolds, one should keep (L1), but replace (L2) by the weaker condition (2), which guarantees that $\{f, s\}(p)$ is determined by the 1-jet of $f$ at $p$. It is also interesting to extend $\operatorname{Vect}_{\pi}(P)$ by adding to it representations up to homotopy, [ELW]. However, for our purposes the class of Poisson vector bundles is already too broad and we do not consider these larger semi-rings.

Remark 2.13 (Terminology). We emphasize that neither the total space of a Poisson vector bundle $E$ nor $E^{*}$ nor their fibers carry a natural Poisson structure. The condition that $E$ is a Poisson vector bundle can be expressed in terms of a certain tensor field with values in $E$ and compatible with the Poisson structure on $P$ (cf., Section 3.3). However, here we prefer to think of this condition as that $\Gamma(E)$ is a Poisson module over $C^{\infty}(P)$.

\subsection{Poisson vector bundles as $\Omega^{1}(P)$-modules.}

Recall that the space of one-forms $\Omega^{1}(P)$ on a Poisson manifold $(P, \pi)$ is a Lie algebra with the bracket

$$
[\alpha, \beta]=-L_{\pi^{\#} \alpha} \beta+L_{\pi{ }_{\beta}} \alpha-d \pi(\alpha, \beta),
$$

where $\alpha$ and $\beta$ are in $\Omega^{1}(P)$. This bracket is a natural extension (via the graded Leibniz identity) of the bracket on closed one-forms locally defined by $[d f, d g]=d\{f, g\}$.

For $\alpha \in \Omega^{1}(P)$ and $s \in \Gamma(E)$ we set

$$
[\alpha, s](p)=\{f, s\}(p),
$$

where $f \in C^{\infty}(P)$ is an arbitrary function function with $d f(p)=\alpha_{p}$. From Proposition 2.2 it is easy to see that in this way we obtain a well-defined action of $\Omega^{1}(P)$ on $\Gamma(E)$. The following result is an immediately consequence of the definition:

Proposition 2.14. The bracket (4) turns $\Gamma(E)$ into a module over the Lie algebra $\Omega^{1}(P)$ such that $[\alpha, s]$ depends only on $\alpha_{p}$ and the 1-jet of $s$ at $p$. Conversely, let a vector bundle $E$ be equipped with a bracket $[]:, \Omega^{1}(P) \times$ $\Gamma(E) \rightarrow \Gamma(E)$ satisfying these conditions or, equivalently, such that

$$
[f \alpha, s]=f[\alpha, s]
$$


and

$$
[\alpha, f s]=f[\alpha, s]+\left(L_{\pi^{\#}} f\right) s
$$

for all one-forms $\alpha$, sections $s$, and smooth functions $f$. Then $E$ is a Poisson vector bundle with $\{f, s\}:=[d f, s]$.

Example 2.15 (Continuation of Example 2.8). Let $G$ be a Poisson Lie group, $G^{*}$ its dual, and $V$ a representation of the Lie algebra $\mathfrak{g}$ of $G$. Let $E=G^{*} \times V$. Identifying $\alpha \in \mathfrak{g}$ with a left-invariant one-form on $G^{*}$, we set $[\alpha, s](p)=\alpha_{p} \cdot s(p)$ for a constant section $s$ and extend this bracket to all one-forms and all sections via (5) and (6). As a result, $E$ becomes a Poisson vector bundle.

Alternatively, we can think of the bracket $[\alpha, \cdot]$ as a vector field on $E$ :

Proposition 2.16. For every $\alpha \in \Omega^{1}(P)$ there exists a unique vector field $Z_{\alpha}$ on the total space of $E$ such that

(1) The projection of $Z_{\alpha}$ to $P$ is equal to $\pi^{\#} \alpha$ and hence the (local) flow $\varphi_{\alpha}^{t}$ of $Z_{\alpha}$ sends fibers of $E$ to fibers of $E$. Furthermore, $\varphi_{\alpha}^{t}$ is fiberwise linear and commutes with the action of the structural group of $E$ incorporated in the definition of $E$ as a Poisson vector bundle $(e . g, \mathrm{U}(n)$ if $E$ is Hermitian).

(2) Let $Z_{\alpha}^{*}$ be the vector field induced by $Z_{\alpha}$ on $E^{*}$. Then $L_{Z_{\alpha}^{*}} s=-[\alpha, s]$, where $s$ on the left hand side is viewed as a fiberwise linear function on $E^{*}$.

(3) $Z_{[\alpha, \beta]}=\left[Z_{\alpha}, Z_{\beta}\right]$ for all $\alpha$ and $\beta$ in $\Omega^{1}(P)$.

(4) If $\alpha$ is closed, $\varphi_{\alpha}^{t}$ preserves the structure of a Poisson vector bundle.

(5) $\varphi_{\alpha}^{t}$ is defined on the same interval of time as the (local) flow of $\pi^{\#} \alpha$.

Proof. The first and the second properties of $Z_{\alpha}$ comprise the definition of this vector field. The third property is equivalent to that $\Gamma(E)$ is a $\Omega^{1}(P)$ module; the fourth property is clear. Finally, the last property follows from the first (see, e.g., the proof of [Gi, Theorem 3.4]). We leave out the details of the proof for the sake of brevity. 
Corollary 2.17. Let $E$ be a Poisson vector bundle over a symplectic manifold $P$. Then $E$ is locally Poisson-trivial, and, as in Example 2.6, $\{f, s\}=$ $\nabla_{X_{f}} s$, where $\nabla$ is a flat connection on $E$. As a consequence, $\operatorname{Vect}_{\pi}(P)$ is in a one-to-one correspondence with $\operatorname{Rep}\left(\pi_{1}(P)\right)$.

Corollary 2.18. Denote by $N_{p}^{*}$ the co-normal Lie algebra to the symplectic leaf through $p \in P$. (By definition, $N_{p}^{*}$ is the dual of the normal space to the leaf through p.) The bracket (4) restricts to a well-defined representation of $N_{p}^{*}$ on $E_{p}$. For all $p$ in the same leaf these representations are equivalent to one another. equivalence.

\subsection{Homogeneous Poisson vector bundles and homotopy}

The class of Poisson vector bundles is too broad for our purposes as the next example indicates. (See also Example 4.7.)

Example 2.19. Let $E$ be an ordinary vector bundle over a trivial Poisson manifold $P$ (i.e., $\pi=0$ ) and let $\sigma$ be a family, parameterized by $p \in P$, of representations of commutative Lie algebras $N_{p}^{*}=T_{p}^{*} P$ on $E_{p}$, i.e., $\sigma \in \Gamma\left(T P \otimes\left(E^{*} \otimes E\right)\right)$. Then $\{f, s\}=\sigma(d f) s$ or, equivalently, $[\alpha, s]=\sigma(\alpha) s$ makes $E$ into a Poisson vector bundle. This Poisson vector bundle is Hermitian if and only if all operators $\sigma(\alpha)$ are anti-self-adjoint with respect to some Hermitian metric on $E$.

We propose two essentially different ways to reduce the class of Poisson vector bundles: by imposing an additional constraint to eliminate undesirable ones and by introducing a new equivalence relation which is weaker than isomorphism.

Definition 2.20. Poisson vector bundles $E_{0}$ and $E_{1}$ over $P$ are deformation equivalent or homotopic if $E_{0}$ and $E_{1}$ are isomorphic to vector bundles that can be connected by a family $E_{t}$ of Poisson vector bundles, smooth in $t \in$ $[0,1]$. This is equivalent to requiring that $E_{0}$ and $E_{1}$ are isomorphic to the restrictions of some Poisson vector bundle $E$ over $P \times[0,1]$ to $P \times\{0\}$ and $P \times\{1\}$, respectively.

We denote the semi-ring of deformation equivalence classes of Poisson vector bundles over $P$ by $\operatorname{Vect}_{\pi}^{\text {def }}(P)$. In the Hermitian or Euclidean case, the vector bundles $E_{t}$ (or, equivalently, $E$ ) are required to be in the same category as $E_{0}$ and $E_{1}$. 
Definition 2.21. A Poisson vector bundle $E$ is homogeneous if every smooth family $\psi_{t}: P \rightarrow P$ of Poisson diffeomorphisms with $\psi_{0}=i d$ admits a lift to a family $\tilde{\psi}_{t}$ of automorphisms of $E$ as a Poisson vector bundle with $\tilde{\psi}_{0}=i d$, i.e.,

$$
\tilde{\psi}_{t}^{*}\{f, s\}=\left\{\psi_{t}^{*} f, \tilde{\psi}_{t}^{*} s\right\}
$$

(These lifts are not a part of the structure of a homogeneous vector bundle.) This is equivalent to that for every Poisson vector field $X$ on $P$, there exists an $\mathbb{R}$-linear operator $D_{X}: \Gamma(E) \rightarrow \Gamma(E)$ such that

$$
D_{X}[\alpha, s]=\left[L_{X} \alpha, s\right]+\left[\alpha, D_{X} s\right] \quad \text { and } \quad D_{X}(f s)=\left(L_{X} f\right) s+f D_{X} s
$$

for all $s \in \Gamma(E)$, all $f \in C^{\infty}(P)$, and all $\alpha \in \Omega^{1}(P)$.

Note that when $\psi_{t}$ is given by a family of closed differential one-forms, the existence of the lift $\tilde{\psi}_{t}$ is guaranteed by Proposition 2.16. The essence of Definition 2.21 is that a homogeneous Poisson vector bundle over $P$ is as homogeneous as $P$ itself. For example, when $E$ is homogeneous, the representations $E_{p}$ of $N_{p}^{*}$ are equivalent to one another for all points $p$ which can be obtained from each other by families of Poisson diffeomorphisms. We denote the semi-ring of isomorphism classes of homogeneous Poisson vector bundles over $P$ by $\operatorname{Vect}_{\pi}^{\text {hom }}(P)$.

Example 2.22. By Corollary 2.17, every Poisson vector bundle over a symplectic manifold $P$ is homogeneous. Two such vector bundles are homotopic if and only if the corresponding representations of $\pi_{1}(P)$ belong to the same path-connected component of $\operatorname{Rep}\left(\pi_{1}(P)\right)$. Hence, $\operatorname{Vect}_{\pi}(P)=$ $\operatorname{Vect}_{\pi}^{\text {hom }}(P)=\operatorname{Rep}\left(\pi_{1}(P)\right)$ and $\operatorname{Vect}_{\pi}^{\mathrm{def}}(P)=\pi_{0}\left(\operatorname{Rep}\left(\pi_{1}(P)\right)\right)$.

Let us now classify Poisson vector bundles over a Poisson manifold with trivial Poisson structure.

Proposition 2.23. Every Poisson vector bundle over a trivial Poisson manifold $P$ arises as described in Example 2.19 and all such vector bundles are homotopic to one another: $\operatorname{Vect}_{\pi}^{\mathrm{def}}(P)=\operatorname{Vect}(P)$. The only homogeneous Hermitian (or Euclidean) Poisson vector bundle over $P$ is the one with the trivial bracket [, ] $=0$ as in Example 2.5.

Proof. The first assertion is clear by Proposition 2.14. If $E$ is homogeneous, the representations $\sigma(p)$ are equivalent to each other for all $p \in P$. Furthermore, by applying diffeomorphisms fixing $p$, we conclude from (7) that the 
operators $\sigma_{p}(\alpha): E_{p} \rightarrow E_{p}, \alpha \in T^{*} P$, are conjugate to each other for all $\alpha \neq 0$ and, hence, have the same eigenvalues. In particular, the operators $\sigma_{p}(\alpha)$ and $\sigma_{p}(a \alpha)$ have the same eigenvalues for all constants $a \neq 0$. Since $\sigma_{p}(a \alpha)=a \sigma_{p}(\alpha)$, these eigenvalues must be zero. This implies that $\sigma_{p}=0$, when $E$ is Hermitian or Euclidean. Hence, $[]=$,0 .

Proposition 2.23 does not extend to all homogeneous vector bundles. In other words, for a Poisson manifold with zero Poisson structure the semi-ring $\operatorname{Vect}_{\pi}^{\text {hom }}(P)$ is in general bigger than $\operatorname{Vect}(P)$.

Example 2.24. Let $P=\mathbb{R}$ and $E=\mathbb{R} \times \mathbb{C}^{n}$. Denote by $A$ the $n \times n$-Jordan block and set $[d x, s]=\sigma(d x) s=A s$, where $x$ is the coordinate on $\mathbb{R}$. We extend the bracket to all one-forms on $P$ by (5). It is easy to see that the resulting Poisson vector bundle is homogeneous.

Proposition 2.25. Let $F$ be a simply connected symplectic manifold, $B$ a Poisson manifold, and let $P=B \times F$ be equipped with the product Poisson structure. Then there is a one-to-one correspondence between Poisson vector bundles over $P$ and Poisson vector bundles over $B$. The same holds for deformation equivalence classes. Thus $\operatorname{Vect}_{\pi}(P) \cong \operatorname{Vect}_{\pi}(B)$ and $\operatorname{Vect}_{\pi}^{\mathrm{def}}(P) \cong \operatorname{Vect}_{\pi}^{\mathrm{def}}(B)$. If the Poisson structure on $B$ is zero, $\operatorname{Vect}_{\pi}^{\text {hom }}(P) \cong \operatorname{Vect}_{\pi}^{\text {hom }}(B)$.

Proof. Proposition 2.25 is a particular case of Propositions 5.9 and 6.5. Let us outline a direct proof, leaving entirely aside the question of homogeneity.

Denote by $p: P=B \times F \rightarrow B$ the natural projection. Fixing the direct product structure on $P$, we embed $C^{\infty}(F)$ into $C^{\infty}(P)$ as a Poisson subalgebra. (Here we use the fact that the symplectic structure on $F_{y}=$ $p^{-1}(y)$ is independent of $y \in B$.) Clearly, for a Poisson vector bundle $E$ over $B$, the pull-back $p^{*} E$ is a Poisson vector bundle over $P$, where the action of $\Omega^{1}(F)$ on $p^{*} E$ is given by the natural flat connection on $p^{*} E$ along the fibers of $p$. It is easy to see that deformation equivalent vector bundles have deformation equivalent pull-backs.

Let us now construct the correspondence inverse to the pull-back.

Let $E$ be a Poisson vector bundle over $P$. The restriction $\left.E\right|_{F_{y}}$ to a fiber $F_{y}$ inherits the structure Poisson vector bundle. Namely, for $h \in C^{\infty}(F)$ and $s \in \Gamma\left(\left.E\right|_{F_{y}}\right)$, we set $\{h, s\}=\left.\{h, \bar{s}\}\right|_{F_{y}}$, where $\bar{s}$ is an arbitrary extension of $s$ to a section of $E$. To verify that this bracket is well defined, we need to show that if $s$ is a section of $E$ vanishing along $F_{y}$ and $h \in C^{\infty}(F)$, then 
$\{h, s\}$ vanishes along $F_{y}$. To this end note that locally $s=\sum f_{j} s_{j}$, where $f_{j}$ are some functions on $P$ vanishing along $F_{y}$ and $s_{j}$ are some sections. Hence,

$$
\{h, s\}=\sum\left(\left\{h, f_{j}\right\} s_{j}+f_{j}\left\{h, s_{j}\right\}\right) .
$$

Clearly, every term on the right hand side vanishes along $F_{y}$ and hence so does the left hand side.

By Corollary 2.17, $\left.E\right|_{F_{y}}$ is a trivial vector bundle with the Poisson vector bundle structure arising from a flat connection. In other words, we obtain a flat $p$-fiberwise connection $\nabla$ on $E$. Let $E_{B}(y)$ be the space of flat sections of $\left.E\right|_{F_{y}}$. The spaces $E_{B}(y)$ form a vector bundle $E_{B}$ over $B$. The sections of $E_{B}$ can be identified with a subspace of $\Gamma(E)$ formed by sections which $\nabla$ horizontal. It is easy to see that $\Gamma\left(E_{B}\right)$ is invariant under the bracket with elements of $p^{*} C^{\infty}(B)$ and thus inherits the structure of a Poisson vector bundle via the embedding $p^{*}: C^{\infty}(B) \rightarrow C^{\infty}(P)$. Therefore, $E=p^{*} E_{B}$. It is clear that the correspondence $E \mapsto E_{B}$ preserves deformation equivalence. This completes the proof of the proposition.

In Sections 5 and 6 we will prove some variations on the theme of Proposition 2.25. Here we mention only the most obvious one.

Proposition 2.26. Let $P$ be a Poisson manifold whose symplectic foliation is a fibration $P \rightarrow B$ with compact and simply connected fibers. Assume also that the cohomology class of the symplectic structure on the fibers does not vary from fiber to fiber. Then there is a one-to-one correspondence between Poisson vector bundles over $P$ and over $B$. The same holds for homogeneous Poisson vector bundles or deformation equivalence classes.

Proof. The argument is similar to the proof of Proposition 2.25 with Moser's theorem used to introduce a local (in $B$ ) direct product structure on $P$.

This result does not generalize to the case where the cohomology class of the symplectic structure on $F$ varies from fiber to fiber; see Example 4.6.

Remark 2.27. In this paper we do not attempt to introduce the "Poisson fundamental group", or groupoid, of a Poisson manifold $P$. However, on the conceptual level, $\operatorname{Vect}_{\pi}(P)$, or rather $\operatorname{Vect}_{\pi}^{\text {hom }}(P)$, should be thought of as the space of representations of such a group be it defined. 


\section{5. $K$-rings.}

Definition 2.28. The homotopy Poisson $K$-ring, $K_{\pi}^{\text {def }}(P)$, of a Poisson manifold $P$ is the Grothendieck rings associated with $\operatorname{Vect}_{\pi}^{\text {def }}(P)$. The homogeneous Hermitian Poisson $K$-ring, $K U_{\pi}^{\text {hom }}(P)$, is the Grothendieck ring associated with the semi-ring of homogeneous Hermitian Poisson vector bundles over $P$. The homogeneous orthogonal Poisson $K$-ring, $K O_{\pi}^{\text {hom }}(P)$, is defined similarly.

We emphasize that $K_{\pi}^{\text {def }}$ denotes one of the four deformation $K$-rings: complex, real, Hermitian, or Euclidean. Likewise, $K$ is either complex (or equivalently, Hermitian) or real (equivalently, orthogonal) ordinary $K^{0}$ functor. In the homogeneous case, we consider only the Hermitian ring $K U_{\pi}^{\text {hom }}$ and the Euclidean one $K O_{\pi}^{\text {hom }}$. In every formula, all $K$-rings are to be understood in the same category. Note that none of the Poisson $K$-rings is functorial with respect to Poisson maps.

From the results of the previous section we conclude that these $K$-rings satisfy (K1) and (K2):

Corollary 2.29. 1. Let, as in Proposition 2.25, $F$ be a simply connected symplectic manifold, $B$ a Poisson manifold, and let $P=B \times F$ be equipped with the product Poisson structure. Then $K_{\pi}^{\mathrm{def}}(P)=K_{\pi}^{\mathrm{def}}(B)$ as rings. If $B$ carries the zero Poisson structure, we also have $K_{\pi}^{\text {def }}(P)=K(B)$ and $K U_{\pi}^{\text {hom }}(P)=K U(B)$.

2. Let $P$ be symplectic. Then $K U_{\pi}^{\text {hom }}(P)=R U\left(\pi_{1}(P)\right)$, where $R U$ stands for the ring of virtual unitary representations, and $K_{\pi}^{\mathrm{def}}(P)$ is the Grothendieck ring generated by $\pi_{0}\left(\operatorname{Rep}\left(\pi_{1}(P)\right)\right)$.

Of course, similar results hold in the Euclidean case.

Example 2.24 readily implies that for the zero Poisson structure the Grothendieck ring associated with $\operatorname{Vect}_{\pi}^{\text {hom }}(P)$ can be strictly greater than $K(P)$, i.e., (K1) fails for this ring. This is the reason that we do not "nominate" the Grothendieck ring of $\operatorname{Vect}_{\pi}^{\text {hom }}(P)$ to be a Poisson $K$-ring. Nor do we consider the Grothendieck ring associated with $\operatorname{Vect}_{\pi}(P)$, for this ring is enormous as Example 2.19 shows.

\section{Representations of Lie Algebroids and Groupoids.}

The goal of this section is to illuminate the connection between Poisson vector bundles and representations of Lie algebroids and groupoids. We 
begin by recalling in Sections 3.1 and 3.2 the necessary definitions and known results important for what follows. We refer the reader to [CdSW, Ma] for a general introduction and original references; additional references are provided in the text as needed. In Section 3.3 we outline the construction of secondary characteristic classes of algebroid representations, due to Crainic, [Cr1], in a way suitable for our exposition and in Section 3.4 we prove that for a homogeneous vector bundle these characteristic classes are invariant under the action of $H_{\pi}^{1}(P)$ on $H_{\pi}^{*}(P)$.

\subsection{Representations of Lie algebroids.}

Let $\mathbb{A} \rightarrow P$ be a Lie algebroid over a manifold $P$ with anchor map $\kappa$ and bracket $[$,$] .$

Definition 3.1. A representation of $\mathbb{A}$ or an $\mathbb{A}$-equivariant vector bundle $E$ over $P$ is a vector bundle $E$ equipped with a bracket $[]:, \Gamma(\mathbb{A}) \times \Gamma(E) \rightarrow$ $\Gamma(E)$ turning $\Gamma(E)$ into a Lie algebra module over $\Gamma(A)$ and such that

$$
[\alpha, s](p) \text { depends only on } \alpha_{p}
$$

and

$$
[\alpha, f s]=\left(L_{\kappa(\alpha)} f\right) s+f[\alpha, s]
$$

for all sections $\alpha$ of $\mathbb{A}$, all sections $s$ of $E$, and all smooth functions $f$ on $P$.

Note that the condition (9) is equivalent to (5). Equation (10) guarantees that $[\alpha, s](p)$ depends only on the 1-jet of $s$ at $p$ and expresses the fact that the bracket [, ] is a lift of $\kappa$ to $E$ via infinitesimal automorphisms of $E$.

Remark 3.2 (Terminology). Calling $E$ a representation of $\mathbb{A}$ is consistent with regarding an algebroid as a generalization of a Lie algebra. The name an "A-equivariant vector bundle" comes from a different perspective: we view an algebroid as a generalization of a Lie algebra action on a manifold; see Example 3.3. The same applies to equivariant vector bundles over groupoids considered later on.

In the terminology of $[\mathrm{Fe} 1, \mathrm{Fe} 2]$, representations of $\mathbb{A}$ are vector bundles with flat connections over $\mathbb{A}$.

Example 3.3. Let $\mathbb{A}$ be the action algebroid $P \times \mathfrak{g}$ for an infinitesimal action of a Lie algebra $\mathfrak{g}$ on $P$. Then a representation of $\mathbb{A}$ is just a $\mathfrak{g}$-equivariant vector bundle over $P$. (See, e.g., $[\mathrm{CdSW}]$ for the definitions.) 
Example 3.4. Let $\mathbb{A}$ be the algebroid of vector fields tangent to a regular foliation $\mathcal{F}$ on $P$. Then a representation of $\mathbb{A}$ is a vector bundle equipped with a partial flat connection along $\mathcal{F}$. An example of such a vector bundle is the normal bundle to $\mathcal{F}$ with the Bott connection.

Remark 3.5. Proposition 2.16, with obvious changes of notations, holds for representations of algebroids.

Remark 3.6 (Holonomy, $[\mathbf{F e} 2]$ ). Let $\gamma:[0,1] \rightarrow \mathbb{A}$ be a mapping with the property that $\kappa(\gamma)=(p \circ \gamma)^{\prime}$, where $p: \mathbb{A} \rightarrow P$ is the projection and the prime denotes the derivative with respect to time. Following Fernandes [Fe2], we refer to such a path as an $\mathbb{A}$-path. There is a well-defined holonomy map $h(\gamma): E_{\gamma(0)} \rightarrow E_{\gamma(1)}$; see [Fe2] (and also [Fe1, GG] in the Poisson setting). This concept arises naturally when we interpret $E$ as a vector bundle with $\mathbb{A}$-connection. (Alternatively, pick a time-dependent section $\alpha_{t}$ of $\mathbb{A}$ such that $\alpha_{t}(p \gamma(t))=\gamma(t)$ and let, in the notation of Proposition 2.16, $\varphi_{t}$ be the time-dependent flow of $Z_{\alpha_{t}}$. Then $h(\gamma)=\left.\varphi^{1}\right|_{E_{\gamma(0)}}$; see [GG].) Holonomy is defined in a setting more general than for representations of $\mathbb{A}$, but only for representations it is in some sense homotopy invariant. We will use this notion in the proof of Theorem 5.3.

Example 3.7. Unless $\kappa=0$, the original bracket [, ] on $\mathbb{A}$ does not make $\mathbb{A}$ a representation of $\mathbb{A}$ on itself: the bracket $[\alpha, s](p)$ of two sections $\alpha$ and $s$ of $\mathbb{A}$ depends on the values of $\alpha$ and $s$ at $p$. In other words, (5) and (9) fail. Likewise, when $P$ is a Poisson manifold, the bracket $\{f, s\}=[d f, s]$, for $f \in C^{\infty}(P)$ and $s \in \Omega^{1}(P)$, satisfies (L1), but not (L2).

Recall that the algebroid $T^{*} P$ is canonically associated to every Poisson manifold $(P, \pi)$; see, e.g., [CdSW, Section 13.3]. As a vector bundle, this algebroid is $T^{*} P$, the anchor map is $\pi^{\#}$, and the bracket is given by (3). As an immediate consequence of Proposition 2.14, we obtain

Corollary 3.8. There is a one-to-one correspondence between Poisson vector bundles over $P$ and representations of $T^{*} P$.

Example 3.9 (The canonical line bundle, [ELW]). The canonical line bundle $\mathbb{L}=\wedge^{n} T^{*} P$, where $n=\operatorname{dim} P$, is a Poisson vector bundle over $P$ with the action of $\alpha \in \Omega^{1}(P)$ on $s \in \Omega^{n}(P)$ given by $L_{\pi \#(\alpha)} s+\langle\pi, d \alpha\rangle s=$ $\alpha \wedge\left(d \iota_{\pi} s\right)$. The canonical line bundle is homogeneous. (Set $D_{X}=L_{X}$ in (8).) Furthermore, $\mathbb{L}$ is trivial as a Poisson line bundle if and only if $P$ is 
unimodular, i.e., $P$ admits a volume form invariant under all Hamiltonian flows.

More generally, for an arbitrary algebroid $\mathbb{A}$, there is a canonical line bundle $Q_{\mathbb{A}}$ introduced in [ELW], which is also a representation of $\mathbb{A}$. These line bundles are important for the definition of the modular class.

Clearly, the definition of deformation equivalence extends to representations of algebroids and groupoids, considered in the next section.

Remark 3.10. The notion of a homogeneous Poisson vector bundle does not readily generalize to representations of algebroids: it appears to be essential that in Definition $2.21 X$ is both a one-cocycle on $T^{*} P$ and a vector field on $P$. For example, as observed in [Fe2, Gi], every section of $\mathbb{A}$ integrates to a (local) flow of algebroid automorphisms on $\mathbb{A}$ and one can then require these flows to "lift" to $E$. However, in the Poisson case this requirement is, in general, weaker than that of Definition 2.21. Indeed, such flows form a group which in many cases is strictly smaller than the connected component of the identity in the group of all Poisson diffeomorphisms of $P$ considered in Definition 2.21. Perhaps, the right criterion for the "correct" generalization is to have Theorem 6.2 hold for the semi-ring of homogeneous vector bundles.

\subsection{Representations of Lie groupoids.}

Let $\mathbb{G}$ be a smooth groupoid over a manifold $P$.

Definition 3.11. A representation of $\mathbb{G}$ or a $\mathbb{G}$-equivariant vector bundle over $P$ is a vector bundle $E$ equipped with linear maps $\Phi(g): E_{x} \rightarrow E_{y}$ for all $g \in \mathbb{G}$, where $x$ and $y$ are the source and, respectively, the target of $g$, such that $\Phi(g h)=\Phi(h) \Phi(g)$ whenever $g h$ is defined. These maps are assumed to depend smoothly on $g$.

Example 3.12. Let $G$ be a Lie group acting on a manifold $P$ and let $\mathbb{G}=$ $P \times G$ be the action groupoid. Similarly to Example 3.3, representations of $\mathbb{G}$ are simply $G$-equivariant vector bundles over $P$.

Let $\mathbb{A}$ be the algebroid of $\mathbb{G}$. Every representation of $\mathbb{G}$ is naturally a representation of $\mathbb{A}$. As in the case of actions of Lie groups, the converse is also true under a suitable simply connectedness hypothesis. The following result is a consequence of, e.g., [MM, Theorem 3.6]. 
Proposition 3.13. Assume that the source-fibers of $\mathbb{G}$ are simply connected. Then every representation of $\mathbb{A}$ integrates to a representation of $\mathbb{G}$. As a consequence, there is a one-to-one correspondence between representations of $\mathbb{A}$ and $\mathbb{G}$.

This proposition is not very hard to prove directly using, for example, the notion of holonomy; see Remark 3.6.

In what follows, we denote the semi-rings of isomorphism classes of representations of $\mathbb{A}$ or $\mathbb{G}$ by $\operatorname{Vect}_{\mathbb{A}}(P)$ and $\operatorname{Vect}_{\mathbb{G}}(P)$. Passing to infinitesimal actions gives rise to a homomorphisms $\operatorname{Vect}_{\mathbb{G}}(P) \rightarrow \operatorname{Vect}_{\mathbb{A}}(P)$, which is an isomorphism under the hypotheses of Proposition 3.13. The resulting Grothendieck rings, for homotopy classes of representations, are denoted by $K_{\mathbb{A}}^{\text {def }}(P)$ and $K_{\mathbb{G}}^{\text {def }}(P)$, respectively. We also assume the other notation conventions of Section 2.5.

With a topological groupoid $\mathbb{G}$ over a manifold $P$, one can associate the classifying space $B \mathbb{G}$. The construction of the space $B \mathbb{G}$ is similar to Milnor's construction of the classifying space $B G$ for a group $G$; see [Bo1, Se].

Example 3.14. Let $\mathbb{G}=P \times G$ be the action groupoid for an action of a group $G$ on a manifold $P$. Then $B \mathbb{G}$ is the homotopy quotient $(P \times E G) / G$, where $E G$ is a contractible space with a free action of $G$ and $B G=E G / G$ is the classifying space for $G$-principal bundles.

An analogue of Borel's construction shows that every representation of $\mathbb{G}$ gives rise to a vector bundle over $B \mathbb{G}$. Thus we have

$$
\operatorname{Vect}_{\mathbb{G}}(P) \rightarrow \operatorname{Vect}(B \mathbb{G})
$$

where $\operatorname{Vect}(B \mathbb{G})$ is the collection of isomorphism classes of ordinary vector bundles over $B \mathbb{G}$.

In general, the map (11) is not a bijection even when $G$ is compact; see [Ol]. However, as is the case with actions of compact groups, for a Hausdorff proper groupoid the cohomology $H^{*}(B \mathbb{G})$ is exactly the space where the characteristic classes of representations of $\mathbb{G}$ belong. In particular, the completion $H^{* *}(B \mathbb{G} ; \mathbb{R})$ is the target space for the $\mathbb{G}$-equivariant Chern character. With the exception of some (important) examples, groupoids are rarely proper (and sometimes not even Hausdorff), and Borel's construction we have just described appears to be of little use when applied directly and naively to non-proper groupoids. (See, e.g., [Bo2] for more elaborate applications of Borel's construction in the non-proper case.) In the non-proper setting, the corresponding $K$-ring is usually defined using non-commutative 
geometry techniques; see [Con1, Con2] and also [He] for an excellent brief introduction. As we have pointed out above, it is unlikely that $K$-rings considered in this paper give meaningful results for seriously non-proper groupoids. (See, however, Section 4.) Yet it would still be interesting to see the exact relation between $K_{\mathbb{G}}^{\text {def }}$ and its "non-commutative" counterparts.

In any case, for a Poisson manifold $P$, the algebroid $T^{*} P$ may not integrate to a groupoid and the constructions of this section apply only when integration is possible. (See, e.g., Section 4.)

\subsection{Secondary characteristic classes.}

The construction of secondary characteristic classes of representations of $\mathbb{A}$ is similar to that of characteristic classes of vector bundles with flat connections (see, e.g., $[\mathrm{KT}]$ ) or $\mathfrak{g}$-structures, $[\mathrm{Fu}]$. In this section we recall the basic definition, focusing on the case where $E$ is trivial, for only this case is used in the subsequent sections. We refer the reader to [Cr1] for a detailed treatment of the general case. (See also [Fe1, Fe2] for a different construction of these characteristic classes and [Cr2] for a comparison of the two methods.) We also restrict our attention to complex vector bundles. After suitable modifications, analogues of the results proved below hold for vector bundles with other structural groups, with $\mathfrak{g} l(n)$ being replaced by the Lie algebra of the structural group.

Let $\mathbb{A}$ be an algebroid over $P$ and $E$ a complex representation of $\mathbb{A}$ which is trivial as an ordinary vector bundle. Fix a trivialization $E=P \times \mathbb{C}^{n}$. Then, for every section $\alpha$ of $\mathbb{A}$, the vertical component of $Z_{\alpha}$ becomes a $\mathfrak{g} l(n)$-valued function on $P$ (see Proposition 2.16 and Remark 3.5). Thus, we obtain a $C^{\infty}(P)$-linear mapping $\Xi: \Gamma(\mathbb{A}) \rightarrow C^{\infty}(P, \mathfrak{g l}(n))$, which we view as an element in $\Gamma\left(\mathbb{A}^{*}\right) \times \mathfrak{g} l(n)$. In other words,

$$
[\alpha, s]=L_{\kappa(\alpha)} s+\Xi(\alpha) s,
$$

where we have identified $\Gamma(E)$ with $C^{\infty}\left(P, \mathbb{C}^{n}\right)$ using the trivialization. It is easy to see that $\Xi$ satisfies the Maurer-Cartan equation

$$
d_{\AA} \Xi+\frac{1}{2}[\Xi, \Xi]_{\text {Lie }}=0,
$$

where $d_{\mathbb{A}}$ is the differential in algebroid cohomology (see, e.g., [CdSW]) and as usual $[\Xi, \Xi]_{\text {Lie }}(\alpha, \beta)=2[\Xi(\alpha), \Xi(\beta)]$ in $\mathfrak{g l}(n)$. (Here and in what follows, we use the subscript "Lie" to distinguish this bracket from the Schouten bracket.) 
Remark 3.15. It is easy to see that every $\Xi$ satisfying the Maurer-Cartan equation determines the structure of a Poisson vector bundle on a trivial vector bundle $E$.

From this remark we immediately obtain

Corollary 3.16. The isomorphism classes of one-dimensional representations of $\mathbb{A}$ on the trivial line bundle are in a one-to-one correspondence with the first algebroid cohomology $H^{1}(\mathbb{A} ; \mathbb{R})$ for real or Hermitian representation and with $H^{1}(\mathbb{A} ; \mathbb{C})$ for complex line bundles.

Let $c$ be a cocycle $c$ of degree $k$ in the Chevalley-Eilenberg complex and let $\Xi_{c}=c \circ \Xi \in \Gamma\left(\wedge^{k} \mathbb{A}^{*}\right)$. The proof of the following proposition is standard; see, e.g., [Cr1, Fu, KT].

Proposition 3.17. The section $\Xi_{c}$ is $d_{\mathbb{A}}$-closed: $d_{\AA} \Xi_{c}=0$. The cohomology class $\left[\Xi_{c}\right] \in H^{k}(\mathbb{A})$ in the algebroid cohomology of $\mathbb{A}$ is determined on the cohomology class of $c$ in $H^{*}(\mathfrak{g l}(n))$ This class depends of the trivialization of $E$. If $P$ is contractible, $\left[\Xi_{c}\right]$ is independent of trivialization. Alternatively, when the trivializations used in the construction of $\Xi$ are unitary and $[c] \in$ $H^{*}(\mathfrak{g l} l(n), \mathfrak{u}(n))$, the class $\left[\Xi_{c}\right]$ is independent of trivialization.

Remark 3.18. The dependence of $\Xi_{c}$ on the trivialization is only up to the pull-backs of the cohomology class of $c$ in $H^{*}(\mathrm{GL}(n))$ by all elements of $[P, \mathrm{GL}(n)]$. This justifies the last assertion of the proposition since the inclusion $\mathrm{U}(n) \hookrightarrow \mathrm{GL}(n)$ is a homotopy equivalence.

Example 3.19. Assume that $P=\mathfrak{g}^{*}$ and $E=\mathfrak{g}^{*} \times \mathbb{C}^{n}$ is a Poisson vector bundle corresponding to a representation of $\mathfrak{g}$ in $\mathbb{C}^{n}$ as in Example 2.7. Since $\mathfrak{g}^{*}$ is simply-connected, the class $\left[\Xi_{c}\right]$ is well defined. Let us describe the characteristic class homomorphism $[c] \mapsto\left[\Xi_{c}\right]$ explicitly. The representation map $\mathfrak{g} \rightarrow \mathfrak{g l}(n)$ gives rise to a homomorphism $H^{*}(\mathfrak{g} l(n)) \rightarrow H^{*}(\mathfrak{g})$. Then $[c] \mapsto\left[\Xi_{c}\right]$ factors as follows

$$
H^{*}(\mathfrak{g} l(n)) \rightarrow H^{*}(\mathfrak{g}) \rightarrow H^{*}\left(\mathfrak{g} ; C^{\infty}\left(\mathfrak{g}^{*}\right)\right)=H_{\pi}^{*}\left(\mathfrak{g}^{*}\right)
$$

In the last identity we use the canonical identification of the cohomology which holds already on the level of complexes; see, e.g., [Co2, Lu]. The same holds for the action algebroid on $\mathfrak{g}^{*}$ associated with the coadjoint action.

As usual, characteristic classes do not determine the representation. For example, vanishing of all characteristic classes, even for $H^{*}(\mathfrak{g l} l(n))$, does 
not guarantee that a representation is trivial; see, e.g., Example 2.24. The secondary characteristic classes defined via elements of $H^{*}(\mathfrak{g} l(n), \mathfrak{u}(n))$ carry even less information than those arising from $H^{*}(\mathfrak{g l} l(n))$. For instance, if the representation is unitary, for $[c] \in H^{*}(\mathfrak{g l}(n), \mathfrak{u}(n))$ all characteristic classes $\left[\Xi_{c}\right]$ vanish and, in fact, these characteristic classes are some obstructions to making a representation unitary.

Remark 3.20 (Rigidity, [Fu]). Most of the characteristic classes defined above are in fact invariants of homotopy equivalence. For the sake of simplicity let us focus again on trivial vector bundles. Denote by $\mathfrak{h}$ the Lie algebra of the structural group. Then $\left[\Xi_{c}\right]$ is an invariant of homotopy of $E$, provided that $[c] \in \operatorname{ker}\left(\operatorname{var}: H^{*}(\mathfrak{h}) \rightarrow H^{*-1}\left(\mathfrak{h} ; \mathfrak{h}^{*}\right)\right),[\mathrm{Fu}]$. For instance, in the Hermitian case, i.e., when $\mathfrak{h}=\mathfrak{s u}(n)$, every class is an invariant of homotopy. For $\mathfrak{h}=\mathfrak{g l}(n)$, the invariants of homotopy are described as follows. Denote by $u_{1}, \ldots, u_{n}$ multiplicative generators of $H^{*}(\mathfrak{g l} l(n))$ so that $\operatorname{deg} u_{k}=2 k-1$. Then $\left[\Xi_{c}\right]$ is an invariant of homotopy equivalence if and only if the factorization of $[c]$ in these generators does not involve $u_{1}$; see $[\mathrm{Fu}]$.

Example 3.21 ([Cr1, ELW]). Let $\mathbb{L}$ be the canonical line bundle of a Poisson manifold $P$; see Example 3.9. Then the characteristic class of $\mathbb{L}$ corresponding to $c=\operatorname{tr}$ is the modular class of $P$. This class is not rigid.

\subsection{Secondary characteristic classes of homogeneous Poisson vector bundles.}

Let now $P$ be a Poisson manifold. A special feature of homogeneous Poisson vector bundles is that their secondary characteristic classes belong to a much smaller space than those of general Poisson vector bundles. Recall that the first Poisson cohomology $H_{\pi}^{1}(P)$ is a Lie algebra (with the bracket induced by the Lie bracket of vector fields) and $H_{\pi}^{*}(P)$ is a module over $H_{\pi}^{1}(P)$.

Proposition 3.22. Assume that $E$ is homogeneous. Then for every cocycle c the cohomology class $\left[\Xi_{c}\right] \in H_{\pi}^{*}(P)$ is $H_{\pi}^{1}(P)$-invariant.

Remark 3.23. Proposition 3.22 does not hold if the homogeneity assumption is dropped. For example, consider a Poisson manifold $P$ with zero Poisson structure. In this case, $H_{\pi}^{k}(P)$ is just the space of $k$-vector fields on $P$ and the $H_{\pi}^{1}(P)$-action on $H_{\pi}^{*}(P)$ is given by the Schouten bracket, [CdSW]. Non-homogeneous Poisson vector bundles over $P$ (Example 2.19) may have characteristic classes which are not $H_{\pi}^{1}(P)$-invariant. 
Proof. In what follows we assume that the vector bundle $E$ is trivial. The proof of the general case requires only superficial changes. In the calculations below we use the sign convention of $[\mathrm{Fu}]$.

Fix a trivialization $E=P \times \mathbb{C}^{n}$ and thus identify sections of $E$ with functions $s \in C^{\infty}\left(P, \mathbb{C}^{n}\right)$. Let $X$ be a Poisson vector field on $P$. Since $E$ is homogeneous, there exists a function $A \in C^{\infty}(P, \mathfrak{g l}(n))$ such that $D_{X} s=L_{X} s+A s$. Combining (8) with (12), we obtain by a straightforward calculation that

$$
\left(L_{X} \Xi\right)(\alpha)=L_{\pi{ }_{\alpha}} A+[\Xi(\alpha), A]_{\text {Lie }}
$$

for any $\alpha \in \Omega^{1}(P)$.

Let now $c$ be a $k$-cocycle on $\mathfrak{g} l(n)$. Consider the $(k-1)$-vector field $b$ on $P$ defined by

$$
b\left(\alpha_{1}, \ldots, \alpha_{k-1}\right)=c\left(A, \Xi\left(\alpha_{1}\right), \ldots, \Xi\left(\alpha_{k-1}\right)\right) .
$$

We complete the proof by showing that $d_{\pi} b=-L_{X} \Xi_{c}$. This equality is verified by the following (also straightforward) calculation.

First recall that

$$
\begin{aligned}
\left(d_{\pi} b\right)\left(\alpha_{1}, \ldots, \alpha_{k}\right)= & \sum_{i<j}(-1)^{i+j-1} b\left(\left[\alpha_{i}, \alpha_{j}\right], \alpha_{1}, \ldots, \widehat{\alpha_{i}}, \ldots, \widehat{\alpha_{j}}, \ldots, \alpha_{k}\right) \\
& +\sum_{l}(-1)^{l} L_{v_{l}} b\left(\alpha_{1}, \ldots, \widehat{\alpha_{l}}, \ldots, \alpha_{k}\right) \\
= & \sum_{i<j}(-1)^{i+j-1} c\left(A, \Xi\left(\left[\alpha_{i}, \alpha_{j}\right]\right), \xi_{1}, \ldots, \widehat{\xi_{i}}, \ldots, \widehat{\xi_{j}}, \ldots, \xi_{k}\right) \\
& +\sum_{l}(-1)^{l} L_{v_{l}} c\left(A, \xi_{1}, \ldots, \widehat{\xi_{l}}, \ldots, \xi_{k}\right),
\end{aligned}
$$

where we set

$$
v_{m}=\pi^{\#} \alpha_{m} \quad \text { and } \quad \xi_{m}=\Xi\left(\alpha_{m}\right) \quad \text { for } \quad m=1, \ldots, k \text {. }
$$

The Maurer-Cartan equation (13) guarantees that

$$
\Xi\left(\left[\alpha_{i}, \alpha_{j}\right]\right)=\left[\xi_{i}, \xi_{j}\right]_{\text {Lie }}+L_{v_{i}} \xi_{j}-L_{v_{j}} \xi_{i}
$$


Hence,

$$
\begin{aligned}
\left(d_{\pi} b\right)\left(\alpha_{1}, \ldots, \alpha_{k}\right)= & \sum_{i<j}(-1)^{i+j-1} c\left(A,\left[\xi_{i}, \xi_{j}\right]_{\text {Lie }}, \xi_{1}, \ldots, \widehat{\xi}_{i}, \ldots, \widehat{\xi}_{j}, \ldots, \xi_{k}\right) \\
& +\sum_{i<j}(-1)^{i+j-1} c\left(A, L_{v_{i}} \xi_{j}, \xi_{1}, \ldots, \widehat{\xi_{i}}, \ldots, \widehat{\xi_{j}}, \ldots, \xi_{k}\right) \\
& -\sum_{i<j}(-1)^{i+j-1} c\left(A, L_{v_{j}} \xi_{i}, \xi_{1}, \ldots, \widehat{\xi}_{i}, \ldots, \widehat{\xi_{j}}, \ldots, \xi_{k}\right) \\
& +\sum_{l}(-1)^{l} L_{v_{l}} c\left(A, \xi_{1}, \ldots, \widehat{\xi}_{l}, \ldots, \xi_{k}\right) .
\end{aligned}
$$

Obviously,

$$
\begin{array}{rl}
L_{v_{l}} & c\left(A, \xi_{1}, \ldots, \widehat{\xi}_{l}, \ldots, \xi_{k}\right) \\
= & c\left(L_{v_{l}} A, \xi_{1}, \ldots, \widehat{\xi_{l}}, \ldots, \xi_{k}\right) \\
& +\sum_{i<l}(-1)^{i-1} c\left(A, L_{v_{l}} \xi_{i}, \xi_{1}, \ldots, \widehat{\xi}_{i}, \ldots, \widehat{\xi}_{l}, \ldots, \xi_{k}\right) \\
& +\sum_{l<j}(-1)^{j} c\left(A, L_{v_{l}} \xi_{j}, \xi_{1}, \ldots, \widehat{\xi}_{l}, \ldots, \widehat{\xi}_{j}, \ldots, \xi_{k}\right) .
\end{array}
$$

By plugging this into (15) and (16), we eliminate these double sums from the expression for $\left(d_{\pi} b\right)\left(\alpha_{1}, \ldots, \alpha_{k}\right)$. Thus, as a result of these cancelations, we obtain

$$
\begin{aligned}
\left(d_{\pi} b\right)\left(\alpha_{1}, \ldots, \alpha_{k}\right)= & \sum_{i<j}(-1)^{i+j-1} c\left(A,\left[\xi_{i}, \xi_{j}\right]_{\text {Lie }}, \xi_{1}, \ldots, \widehat{\xi}_{i}, \ldots, \widehat{\xi}_{j}, \ldots, \xi_{k}\right) \\
& +\sum_{l}(-1)^{l} c\left(L_{v_{l}} A, \xi_{1}, \ldots, \widehat{\xi}_{l}, \ldots, \xi_{k}\right) .
\end{aligned}
$$

By (14), we have $L_{v_{l}} A=\left(L_{X} \Xi\right)\left(\alpha_{l}\right)-\left[\xi_{l}, A\right]_{\text {Lie }}$. Therefore,

$$
\begin{aligned}
& \left(d_{\pi} b\right)\left(\alpha_{1}, \ldots, \alpha_{k}\right) \\
& =\sum_{i<j}(-1)^{i+j-1} c\left(A,\left[\xi_{i}, \xi_{j}\right]_{\text {Lie }}, \xi_{1}, \ldots, \widehat{\xi}_{i}, \ldots, \widehat{\xi}_{j}, \ldots, \xi_{k}\right) \\
& \quad+\sum_{l}(-1)^{l-1} c\left(\left[A, \xi_{l}\right]_{\text {Lie }}, \xi_{1}, \ldots, \widehat{\xi}_{l}, \ldots, \xi_{k}\right) \\
& \quad+\sum_{l}(-1)^{l} c\left(\left(L_{X} \Xi\right)\left(\alpha_{l}\right), \xi_{1}, \ldots, \widehat{\xi}_{l}, \ldots, \xi_{k}\right) .
\end{aligned}
$$


The first two terms in this formula add up to zero because $c$ is a cocycle on $\mathfrak{g} l(n)$. Hence,

$$
\begin{aligned}
\left(d_{\pi} b\right)\left(\alpha_{1}, \ldots, \alpha_{k}\right) & =\sum_{l}(-1)^{l} c\left(\left(L_{X} \Xi\right)\left(\alpha_{l}\right), \xi_{1}, \ldots, \widehat{\xi}_{l}, \ldots, \xi_{k}\right) \\
& =-\left(L_{X} \Xi_{c}\right)\left(\alpha_{1}, \ldots, \alpha_{k}\right) .
\end{aligned}
$$

\section{Poisson vector bundles over $\mathfrak{g}^{*}$.}

In this section we study Poisson vector bundles on the dual space $\mathfrak{g}^{*}$ of a Lie algebra $\mathfrak{g}$ in connection with the requirement (K3). In particular, we prove that every such vector bundle is homotopic to the one associated with a representation of $\mathfrak{g}$ as in Example 2.7. Furthermore, we prove some results suggesting that for semisimple Lie algebras, every homogeneous Hermitian Poisson vector bundle is isomorphic to the one associated with a representation.

\subsection{Classification up to homotopy.}

Let $E \rightarrow \mathfrak{g}^{*}$ be a Poisson vector bundle. Denote by $V$ the representation of $\mathfrak{g}=N_{0}^{*}$ on $E_{0}$ as in Corollary 2.18. Then as is shown in Example 2.7, the trivial bundle $\mathfrak{g}^{*} \times V$ is a Poisson vector bundle.

Theorem 4.1. The Poisson vector bundles $E$ and $\mathfrak{g}^{*} \times V$ are homotopic.

Proof. The argument is by "pushing the non-linearity of $E$ to infinity" as one does to find a deformation between a diffeomorphism $\mathbb{R}^{n} \rightarrow \mathbb{R}^{n}$ and a linear map.

Pick a trivialization of $E$ so as to identify $E$ with $\mathfrak{g}^{*} \times V$ as ordinary, not Poisson, vector bundles. With the trivialization fixed, the structure of a Poisson vector bundle on $E$ is given by the $\mathfrak{g} l(n)$-valued vector field $\Xi: \Omega^{1}\left(\mathfrak{g}^{*}\right) \rightarrow C^{\infty}\left(\mathfrak{g}^{*}, \mathfrak{g l}(n)\right)$ on $\mathfrak{g}^{*}$ satisfying the Maurer-Cartan equation (13) as described in Section 3.3.

Using the structure of a linear space on $\mathfrak{g}^{*}$, we write

$$
\Xi=\Xi_{0}+R
$$

where $\Xi_{0}$ is the value of $\Xi$ at the origin. The constant $\mathfrak{g l}(n)$-valued vector field $\Xi_{0}$ on $\mathfrak{g}^{*}$ also satisfies the Maurer-Cartan equation. In fact, $\Xi_{0}$ deter- 
mines the Poisson vector bundle $\mathfrak{g}^{*} \times V$ associated with the representation $V$. Note that the Maurer-Cartan equations for $\Xi$ and $\Xi_{0}$ imply that

$$
[\pi, R]+\left[\Xi_{0}, R\right]_{\text {Lie }}+\frac{1}{2}[R, R]_{\text {Lie }}=0
$$

where $\pi$ is the Poisson structure on $\mathfrak{g}^{*}$.

To prove the theorem it suffices to connect $\Xi=\Xi_{1}$ and $\Xi_{0}$ by a family of vector fields $\Xi_{t}, t \in[0,1]$, satisfying the Maurer-Cartan equation (see Remark 3.15). Denote by $\varphi^{t}: \mathfrak{g}^{*} \rightarrow \mathfrak{g}^{*}$ the dilation by factor $t^{-1}$, i.e., $\varphi^{t}(x)=$ $t^{-1} x$ for $x \in \mathfrak{g}^{*}$ and $t \in(0,1]$. Let us show that $\Xi_{t}=t \varphi_{*}^{t} \Xi$ satisfies the Maurer-Cartan equation.

To this end we first observe that $\varphi_{*}^{t} \Xi_{0}=t^{-1} \Xi_{0}$ since $\Xi_{0}$ is a constant vector field. Hence $\Xi_{t}=\Xi_{0}+t \varphi_{*}^{t} R$. Likewise, $\varphi_{*}^{t} \pi=t^{-1} \pi$, for $\pi$ is linear. Therefore,

$$
\begin{aligned}
{\left[\pi, \Xi_{t}\right]+\frac{1}{2}\left[\Xi_{t}, \Xi_{t}\right]_{\text {Lie }} } & =\left[\pi, \Xi_{0}+t \varphi_{*}^{t} R\right]+\frac{1}{2}\left[\Xi_{0}+t \varphi_{*}^{t} R, \Xi_{0}+t \varphi_{*}^{t} R\right]_{\text {Lie }} \\
& =t\left[\pi, \varphi_{*}^{t} R\right]+t\left[\Xi_{0}, \varphi_{*}^{t} R\right]_{\text {Lie }}+\frac{t^{2}}{2}\left[\varphi_{*}^{t} R, \varphi_{*}^{t} R\right]_{\text {Lie }} \\
& =t^{2}\left[\varphi_{*}^{t} \pi, \varphi_{*}^{t} R\right]+t^{2}\left[\varphi_{*}^{t} \Xi_{0}, \varphi_{*}^{t} R\right]_{\text {Lie }}+\frac{t^{2}}{2}\left[\varphi_{*}^{t} R, \varphi_{*}^{t} R\right]_{\text {Lie }} \\
& =t^{2} \varphi_{*}^{t}\left([\pi, R]+\left[\Xi_{0}, R\right]_{\text {Lie }}+\frac{1}{2}[R, R]_{\text {Lie }}\right) \\
& =0
\end{aligned}
$$

Here the second equality follows from that $\Xi_{0}$ satisfies the Maurer-Cartan equation and the last one is a consequence of (17).

Finally, observe that $\Xi_{t}$ extends smoothly in $t$ to $t=0$ with value $\Xi_{0}$ and hence $\Xi_{t}, t \in[0,1]$, is the required homotopy between $\Xi=\Xi_{1}$ and $\Xi_{0}$.

Clearly, Poisson vector bundles associated with representations in the same path-connected component of $\operatorname{Rep}(\mathfrak{g})$ are homotopy equivalent. As a result, we conclude that the requirement (K3) holds for $K_{\pi}^{\text {def }}$ :

Corollary 4.2. For any Lie algebra, $\operatorname{Vect}_{\pi}^{\text {def }}\left(\mathfrak{g}^{*}\right)=\pi_{0}(\operatorname{Rep}(\mathfrak{g}))$ and, hence, $K_{\pi}^{\text {def }}\left(\mathfrak{g}^{*}\right)$ is the Grothendieck ring generated by $\pi_{0}(\operatorname{Rep}(\mathfrak{g}))$. In particular, if $\mathfrak{g}$ is semi-simple, $K_{\pi}^{\text {def }}\left(\mathfrak{g}^{*}\right)=R(\mathfrak{g})$. 


\subsection{Semisimple Lie algebras.}

Throughout this section $G$ is a semisimple simply connected Lie group and $\mathfrak{g}$ is the Lie algebra of $G$.

Theorem 4.3. Let $\mathfrak{g}$ be semisimple of compact type and let $P \subset \mathfrak{g}^{*}$ be a $G$-invariant open set. ${ }^{3}$ Then there is a one-to-one correspondence between Poisson vector bundles over $P$ and $G$-equivariant vector bundles over $P$. Every Poisson vector bundle over $P$ is homogeneous and homotopic vector bundles are isomorphic. Thus, $\operatorname{Vect}_{\pi}(P)=\operatorname{Vect}_{\pi}^{\text {hom }}(P)=\operatorname{Vect}_{\pi}^{\text {def }}(P)=$ $\operatorname{Vect}_{G}(P)$.

Proof. Since $\mathfrak{g}$ is a semisimple Lie algebra of compact type, $\mathfrak{g}$ is the Lie algebra of a compact simply connected Lie group $G$. The algebroid $T^{*} P$ is isomorphic to the action algebroid of the coadjoint action on $P$. The latter integrates to the action groupoid $P \times G$. By Proposition 3.13, there is a one-to-one correspondence between Poisson and $G$-equivariant vector bundles over $P$. Since $G$-equivariant vector bundles are rigid, homotopic vector bundles are isomorphic. As is shown in [GW] (or in [Co2] for closed balls centered at $0 \in \mathfrak{g}^{*}$ ), every Poisson vector field on $P$ is Hamiltonian. Hence, a Poisson vector bundle over $P$ is automatically homogeneous.

Remark 4.4. All assertions of Theorem 4.3 fail for arbitrary Lie algebras; see, e.g., Example 2.19 (with $P=\mathfrak{g}^{*}$ ) for $\mathfrak{g}=\mathbb{R}$. Moreover, as we will show later in this section the theorem fails for semisimple Lie algebras of non-compact type.

Corollary 4.5. Under the hypotheses of Theorem 4.3,

$$
K U_{\pi}^{\text {hom }}(P)=K_{\pi}^{\text {def }}(P)=K_{G}(P),
$$

which is also equal to the Grothendieck ring of $\operatorname{Vect}_{\pi}(P)$. (In the real case, one should replace $K U_{\pi}^{\text {hom }}$ by $K O_{\pi}^{\text {hom }}$.)

An interesting consequence of these results is that the Poisson $K$-rings $K U_{\pi}^{\text {hom }}$ and $K_{\pi}^{\text {def }}$ really depend on the Poisson structure, but not only on the symplectic foliation.

\footnotetext{
${ }^{3}$ To circumvent the difficulties arising in dealing with (equivariant) vector bundles over non-compact spaces, we assume here that $P$ is (equivariantly) homotopy equivalent to a finite $(G)$ - CW-complex. The same applies to other examples where (equivariant) vector bundles over non-compact manifolds are considered.
} 
Example 4.6 (Genuine dependence on Poisson structures). Consider $P=\mathfrak{s} u(2)^{*} \backslash\{0\}$. In other words, $P=S^{2} \times(0, \infty)$ with the foliated symplectic structure depending linearly on $t \in(0, \infty)$. By Corollary 4.5, $K U_{\pi}^{\text {hom }}(P)=K_{\pi}^{\text {def }}(P)=K_{\mathrm{SU}(2)}\left(S^{2}\right)$. This ring is $R U(\mathrm{U}(1))$, i.e., the ring of Laurent polynomials in one variable of degree two. On the other hand, for the product Poisson structure on $S^{2} \times(0, \infty)$, which has the same symplectic foliation, $K U_{\pi}^{\text {hom }}(P)=K_{\pi}^{\text {def }}(P)=K(\mathbb{R})=\mathbb{Z}$, by Corollary 2.29.

The question of classification of Poisson vector bundles over $\mathfrak{g}^{*}$ for a semisimple $\mathfrak{g}$ of non-compact type is much more subtle. For instance, since every one-dimensional representation of $G$ is trivial, an analogue of Theorem 4.3 would imply that every Poisson line bundle over $\mathfrak{g}^{*}$ is Poisson-trivial which is not true as the following example shows.

Example 4.7. By Corollary 3.16, Poisson line bundles over $\mathfrak{g}^{*}$ are classified by $H_{\pi}^{1}\left(\mathfrak{g}^{*}\right)=H^{1}\left(\mathfrak{g} ; C^{\infty}\left(\mathfrak{g}^{*}\right)\right)$. This cohomology is infinite-dimensional when $\mathfrak{g}$ is not of compact type, [We1]. Hence, the collection of isomorphism classes of such line bundles is infinite. Furthermore, combining this construction with that of Example 2.24, one should be able to construct homogeneous Poisson vector bundles over $\mathfrak{g}^{*}$ which do not correspond to representations. However, as in Example 2.24, these vector bundles are not Hermitian.

Conjecture 4.8. Let, as above, $\mathfrak{g}$ be a semisimple Lie algebra of noncompact type.

1. Every homogeneous Hermitian Poisson vector bundle over $\mathfrak{g}^{*}$ is trivial as a Poisson vector bundle.

2. There is a one-to-one correspondence between germs of real analytic Poisson vector bundles over $\mathfrak{g}^{*}$ and representations of $G$.

This first part of this conjecture is essentially based on the lack of examples. Note that since $G$ has no non-trivial finite-dimensional unitary representations, the conjecture implies that there is a one-to-one correspondence between homogeneous Hermitian Poisson vector bundles over $\mathfrak{g}^{*}$ and finite-dimensional unitary representations of $G$. Here the only additional evidence is Proposition 3.22 combined with another conjecture, namely, that $H_{\pi}^{*}\left(\mathfrak{g}^{*}\right)$ contains no non-zero $H_{\pi}^{1}\left(\mathfrak{g}^{*}\right)$-invariant elements.

The situation is more satisfactory in the real analytic setting. First recall that the germ of a real analytic Poisson vector field on $\mathfrak{g}^{*}$ is Hamiltonian with a real analytic Hamiltonian, [Co1, Co3]. By Corollary 3.16, this implies 
that Conjecture 4.8 holds for real analytic line bundles (real, Hermitian, or complex). Furthermore, the following result confirms the conjecture in the category of formal power series:

Theorem 4.9. Every formal vector bundle over $\mathfrak{g}^{*}$ is formally equivalent to the one associated with a representation of $\mathfrak{g}$.

Proof. The argument is similar to the proof of the Poincaré normal form of a vector field and to Weinstein's proof of the formal linearization theorem for Poisson structures, [We1]. Fix a (formal) trivialization of $E$. We write $\Xi$ as a formal power series on $\mathfrak{g}^{*}$ and assume that this formal power series contains only a zero order term and terms of degree greater than or equal to $r>0$ :

$$
\Xi=\Xi_{0}+\Xi_{r}+\cdots \text {. }
$$

Here, as in the proof of Theorem 4.1, the zero order term $\Xi_{0}$ is associated with the representation of $\mathfrak{g}=N_{0}^{*}$ on $E_{0}$ and $\Xi_{r}$ is a $\mathfrak{g l}(n)$-valued vector field on $\mathfrak{g}^{*}$ whose coefficients are homogeneous polynomials of degree $r$. As usual, the dots denote terms of degree higher than $r$.

The vector field $\Xi_{r}$ can be interpreted as a cochain on $\mathfrak{g}$ with coefficients in $S^{r} \mathfrak{g} \otimes \mathfrak{g l}(n)$. Denote by $d$ the differential $C^{1}\left(\mathfrak{g} ; S^{r} \mathfrak{g} \otimes \mathfrak{g} l(n)\right) \rightarrow C^{2}\left(\mathfrak{g} ; S^{r} \mathfrak{g} \otimes\right.$ $\mathfrak{g l}(n))$, where $\mathfrak{g} l(n)$ is turned into a $\mathfrak{g}$-module via $\Xi_{0}$. The Maurer-Cartan equation (13) implies that

$$
d \Xi_{r}=d_{\pi} \Xi_{r}+\left[\Xi_{r}, \Xi_{0}\right]_{\text {Lie }}=0
$$

i.e., $\Xi_{r}$ is a cocycle.

Our goal is to find a formal change of trivialization $\Phi_{r}$ of $E$ which eliminates the $r$-th order term $\Xi_{r}$. We look for $\Phi_{r}$ in the form $i d+A_{r}$, where $A_{r}$ is a homogeneous $\mathfrak{g l}(n)$-valued polynomial on $\mathfrak{g}^{*}$ of degree $r$. (In the Hermitian case, $A_{r}$ is $\mathfrak{u}(n)$-valued and $\Phi_{r}=\exp A_{r}$.) Under any formal change of trivialization $\Phi: \mathfrak{g}^{*} \rightarrow \mathrm{GL}(n)$, the $\mathfrak{g l}(n)$-valued vector field $\Xi$ transforms to $\Phi^{*} \Xi=\Phi \Xi \Phi^{-1}+\left(d_{\pi} \Phi\right) \Phi^{-1}$. Hence,

$$
\Phi_{r}^{*} \Xi=\Xi_{0}+\left(\Xi_{r}+\left[A_{r}, \Xi_{0}\right]_{\text {Lie }}+d_{\pi} A_{r}\right)+\cdots .
$$

In other words, to eliminate $\Xi_{r}$, we need to find $A_{r}$ with $d A_{r}=-\Xi_{r}$. Since $\mathfrak{g}$ is semisimple, $H^{1}\left(\mathfrak{g} ; S^{r} \mathfrak{g} \otimes \mathfrak{g} l(n)\right)=0$ and, by (18), $A_{r}$ does exist.

To eliminate all non-constant terms in the power series $\Xi$, we argue inductively. Let $\Phi_{1}$ be a change of trivialization with $d A_{1}=-\Xi_{1}$ and $\Phi_{2}$ a change of trivialization eliminating the second order terms in the resulting 
power series, etc. Then the composition $\Phi_{r} \circ \cdots \circ \Phi_{1}$ eliminates all terms up to degree $r+1$. Furthermore, the terms of any given degree in the expansion of $\Phi_{r} \circ \cdots \circ \Phi_{1}$ stabilize as $r \rightarrow \infty$. Hence, $\Phi=\lim _{r \rightarrow \infty} \Phi_{r} \circ \cdots \circ \Phi_{1}$ is well defined as a formal power series. This is the required change of trivialization.

Remark 4.10. It appears plausible that by using the method of majorants as in [Co1, Co3] one can control convergence of the power series $\Phi$ and extend the theorem to the real analytic case, thus proving the second part of Conjecture 4.8.

\section{Morita Invariance of $K$-rings.}

\subsection{Morita equivalence.}

Recall that two Poisson manifolds $\left(P_{1}, \pi_{1}\right)$ and $\left(P_{2}, \pi_{2}\right)$ are Morita equivalent if there exists a symplectic manifold $W$ and submersions

$$
P_{1} \stackrel{\rho_{1}}{\leftarrow} W \stackrel{\rho_{2}}{\rightarrow} P_{2}
$$

with simply connected fibers such that $\rho_{1}$ is Poisson, $\rho_{2}$ is anti-Poisson, and the fibers of $\rho_{1}$ and $\rho_{2}$ are symplectic orthogonal to each other, [Xu1]. ${ }^{4}$ (Note that here and in what follows all submersions are assumed to be surjective.) The essence of this definition is that a Morita equivalence between Poisson manifolds gives rise to a bijection between their symplectic leaves and corresponding leaves have equal first cohomology and anti-isomorphic normal Poisson structures.

Let us summarize the properties of Morita equivalence which are important for what follows. (In (M1), except the last assertion, and (M2) it suffices to assume that $\rho_{1^{-}}$and $\rho_{2}$-fibers are connected.)

(M1) [We1]. For a symplectic leaf $F \subset P_{1}$, the projection $\rho_{2}\left(\rho_{1}^{-1}(F)\right)$ is a symplectic leaf in $P_{2}$. By symmetry, $F \mapsto \rho_{2}\left(\rho_{1}^{-1}(F)\right)$ is a oneto-one correspondence between symplectic leaves of $P_{1}$ and $P_{2}$. The corresponding leaves have anti-isomorphic normal Poisson structures and isomorphic first cohomology groups.

\footnotetext{
${ }^{4}$ The original definition of Morita equivalence given in [Xu1] requires the projections $\rho_{1}$ and $\rho_{2}$ to be complete, [We1]. Following a suggestion of Alan Weinstein, we omit this additional assumption, for completeness is immaterial for the properties of Morita equivalence discussed below and for other results proved in this section, cf., [GG, Remark 4.7].
} 
(M2) [We1]. The Poisson annihilator of $\rho_{1}^{*} C^{\infty}\left(P_{1}\right)$ in $C^{\infty}(W)$ is $\rho_{2}^{*} C^{\infty}\left(P_{2}\right)$, and vice versa. The manifolds $P_{1}$ and $P_{2}$ have equal spaces of Casimir functions, both isomorphic to $\rho_{1}^{*} C^{\infty}\left(P_{1}\right) \cap \rho_{2}^{*} C^{\infty}\left(P_{2}\right)$.

(M3) [GL]. Morita equivalent Poisson manifolds $P_{1}$ and $P_{2}$ have isomorphic first Poisson cohomology spaces. More explicitly, there is a natural isomorphism

$$
I_{W}: H_{\pi}^{1}\left(P_{1}\right) \stackrel{\cong}{\rightrightarrows} H_{\pi}^{1}\left(P_{2}\right),
$$

which is equal, up to a sign, to the one found in [Cr1]. We will recall the definition of this isomorphism in Lemma 5.7.

Remark 5.1. Morita equivalence of Poisson manifolds is closely connected with the notion of Morita equivalence of symplectic groupoids, studied by Ping $\mathrm{Xu},[\mathrm{Xu} 1, \mathrm{Xu} 2]$, and topological groupoids, [MRW]. In fact, for integrable Poisson manifolds (i.e., those which admit global symplectic groupoids), it is equivalent to Morita equivalence of symplectic groupoids. In Section 6 we consider an infinitesimal version of Morita equivalence of groupoids.

Remark 5.2. In spite of its name, Morita equivalence of Poisson manifolds is not an equivalence relation. However, it becomes such when restricted the class of integrable Poisson manifolds; see [Xu2] for more details. For non-integrable Poisson manifolds, the full meaning of Morita equivalence is not quite clear, yet in what follows we make no integrability assumptions.

\subsection{Morita equivalence and $K$-rings.}

Theorem 5.3. A Morita equivalence (19) induces an isomorphism between semi-rings of Poisson vector bundles on $P_{1}$ and $P_{2}$, [Cr1], homotopy equivalence classes of such vector bundles, and semi-rings of homogeneous vector bundles:

$$
\begin{aligned}
\operatorname{Vect}_{\pi}\left(P_{1}\right) & \cong \operatorname{Vect}_{\pi}\left(P_{2}\right), \quad[\mathrm{Cr} 1], \\
\operatorname{Vect}_{\pi}^{\text {def }}\left(P_{1}\right) & \cong \operatorname{Vect}_{\pi}^{\text {def }}\left(P_{2}\right), \\
\operatorname{Vect}_{\pi}^{\text {hom }}\left(P_{1}\right) & \cong \operatorname{Vect}_{\pi}^{\text {hom }}\left(P_{2}\right) .
\end{aligned}
$$

Corollary 5.4. $K_{\pi}^{\text {def }}\left(P_{1}\right)=K_{\pi}^{\text {def }}\left(P_{2}\right)$ and $K U_{\pi}^{\text {hom }}\left(P_{1}\right)=K U_{\pi}^{\text {hom }}\left(P_{2}\right)$. 
The proof of (21) is given in [Cr1] in the integrable case and a more general infinitesimal argument is also outlined there. Then (22) is an immediate consequence of the proof. Let us recall some elements of the proof which are needed to verify (23) and also used in Section 6.

We begin by recalling the notion of an inverse-image or pull-back or induced algebroid, [HM, p. 202-203]. Let $\rho: Q \rightarrow P$ be a submersion and let $\mathbb{A}$ be an algebroid over $P$ with anchor $\kappa$. As a vector bundle, the inverseimage algebroid $\rho^{\star} \mathbb{A}$ is a subbundle in $\rho^{*} \mathbb{A} \oplus T Q$ formed by pairs $(\alpha, X)$ with $\kappa(\alpha)=\rho_{*} X$ (We reserve the notation $\rho^{\star} \mathbb{A}$ for the inverse-image algebroid to distinguish it from the pull-back vector bundle $\rho^{*} \mathbb{A}$.) Note that $\operatorname{ker} \rho_{*}$ is a subalgebroid in $\rho^{\star} \mathbb{A}$ and $\rho^{\star} \mathbb{A} \cong \rho^{*} \mathbb{A} \oplus \operatorname{ker} \rho_{*}$ as vector bundles. The bracket on $\rho^{\star} \mathbb{A}$ is given by

$$
[(f \alpha, X),(g \beta, Y)]=\left(f g[\alpha, \beta]+\left(L_{X} f\right) \alpha-\left(L_{Y} g\right) \beta,[X, Y]\right),
$$

where $f$ and $g$ are smooth functions on $Q, \alpha$ and $\beta$ are pull-backs of sections of $\mathbb{A}$, and $X$ and $Y$ are vector fields on $Q$. The anchor map on $\rho^{\star} \mathbb{A}$ is simply $(\alpha, X) \mapsto X$.

Example 5.5. The pull-back $\rho^{\star} T P$ is naturally isomorphic to $T Q$ with the isomorphism being the anchor map. This is easy to check in local coordinates.

For a representation $E$ of $\mathbb{A}$, its pull-back $\rho^{*} E$ is automatically a representation of $\rho^{\star} \mathbb{A}$ such that, in the notations of the above formula,

$$
[(\alpha, X), f s]=\left(L_{X} f\right) s+f[\alpha, s]
$$

where $s$ is the pull-back of a section of $E,[\mathrm{HM}]$.

Lemma 5.6 ([Cr1]). Let the fibers of $\rho$ be $l$-connected with $l \geq 1$. Then the pull-back $E \mapsto \rho^{*} E$ induces a bijection

$$
\operatorname{Vect}_{\mathbb{A}}(P) \stackrel{\cong}{\rightarrow} \operatorname{Vect}_{\rho^{\star}}(Q) .
$$

Furthermore, the natural pull-back homomorphism $\rho^{*}: H^{k}(\mathbb{A}) \rightarrow H^{k}\left(\rho^{\star} \mathbb{A}\right)$ is an isomorphism for $k \leq l$.

The first assertion readily follows from the fact that the $\operatorname{ker} \rho_{*}$-action on $\rho^{*} E$ coincides with the one arising from the natural flat connection on $\rho^{*} E$ along $\rho$-fibers. The second assertion is proved in [Cr1] by using an analogue of the Serre-Hochschild spectral sequence (see, e.g., [Fu]) for algebroids, with the subalgebroid $\operatorname{ker} \rho_{*}$. 
Proof of Theorem 5.3. Let us set $\mathbb{A}_{1}=T^{*} P_{1}$ and $\mathbb{A}_{2}=T^{*} P_{2}$. By (24), to establish (21), it suffices to show that $\rho_{1}^{\star} \mathbb{A}_{1}$ and $\rho_{2}^{\star} \mathbb{A}_{2}$ are anti-isomorphic. The required anti-isomorphism $J: \rho_{1}^{\star} \mathbb{A}_{1} \rightarrow \rho_{2}^{\star} \mathbb{A}_{2}$ is

$$
J:(\alpha, X) \mapsto\left(\iota_{X} \omega-\alpha, X\right)
$$

where $\omega$ is the symplectic structure on $W$. Combined with (24), this proves (21). Moreover, it is clear that the bijection (24) induces a bijection on the level of homotopy equivalence classes thus giving (22). Furthermore, (21) preserves the classes of Hermitian or Euclidean vector bundles.

To prove (23), we will show that the bijection (21) sends homogeneous vector bundles to homogeneous ones. We need the following fact, which is implicitly contained in [GL] and explicitly in [GG]:

Lemma 5.7 ([GG, GL]). Let $\xi_{1}$ be Poisson vector fields on $P_{1}$. Then there exists a Hamiltonian vector field $\xi$ on $W$ and a Poisson vector field $\xi_{2}$ on $P_{2}$ such that

$$
\left(\rho_{1}\right)_{*} \xi=\xi_{1} \quad \text { and } \quad\left(\rho_{2}\right)_{*} \xi=-\xi_{2}
$$

Furthermore, $I_{W}\left(\left[\xi_{1}\right]\right)=\left[\xi_{2}\right]$.

Let $E^{(1)}$ be a homogeneous Poisson vector bundle over $P_{1}$ and let $E^{(2)}$ be a Poisson vector bundle over $P_{2}$ which corresponds to $E^{(1)}$ under (21). Denote by $E$ the vector bundle $\rho_{1}^{*} E^{(1)}=\rho_{2}^{*} E^{(2)}$ over $W$.

Let $\xi_{1}$ be a Poisson vector field on $P_{1}$. Denote by $\psi_{1}$ the (local) flow of $\xi_{1}$ in some time $t$, by $\tilde{\psi}_{1}$ its lift to $E^{(1)}$, and by $\psi$ the (local) flow of $\xi$ in time $t$ on $W$. The flow $\psi$ lifts to $E$. Indeed, for $x$ and $y$ in $W$ such that $\psi(x)=y$, we set

$$
\tilde{\psi}: E_{x} \cong E_{\rho_{1}(x)}^{(1)} \stackrel{\tilde{\psi}_{1}}{\rightarrow} E_{\rho_{1}(y)}^{(1)} \cong E_{y}
$$

(Here we use the fact that $\rho_{1} \psi=\psi_{1}$.) It is easy to see that $\tilde{\psi}$ is indeed a lift of $\psi$ via automorphisms of the representation $E$ of $\rho_{1}^{\star} \mathbb{A}_{1}$, which covers, in the obvious sense, the lift $\tilde{\psi}_{1}$.

By Lemma 5.7, $\psi$ projects to the flow $\psi_{2}$ of $-\xi_{2}$ on $P_{2}$. To complete the proof of (23) we only need to show that $\tilde{\psi}$ has a well-defined projection $\tilde{\psi}_{2}$ which is a lift of $\psi_{2}$ to $E^{(2)}$. Let $x$ and $x^{\prime}$ be two points in the same $\rho_{2}$-fiber. Then for $y=\psi(x)$ and $y^{\prime}=\psi\left(x^{\prime}\right)$ we also have $\rho_{2}(y)=\rho_{2}\left(y^{\prime}\right)$. Our goal is 
to show that the following diagram is commutative:

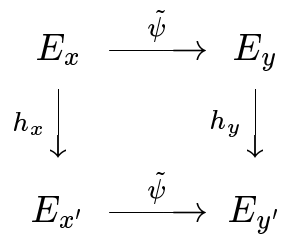

where the vertical arrows are the identifications

$$
h_{x}: E_{x} \cong E_{\rho_{2}(x)}^{(2)} \cong E_{x^{\prime}} \text { and } h_{y}: E_{y} \cong E_{\rho_{2}(y)}^{(2)} \cong E_{y^{\prime}} .
$$

These identifications can also be interpreted in terms of holonomy. Connect $x$ and $x^{\prime}$ by a path $\gamma$ in the $\rho_{2}$-fiber. Then $h_{x}$ is just the holonomy along $\gamma$ with respect to the natural flat $\rho_{2}$-fiberwise connection on $E$. On the other hand, $\gamma$ can also be thought of as a $\operatorname{ker}\left(\rho_{2}\right)_{*}$-path and hence as a $\rho_{2}^{\star} \mathbb{A}_{2}$-path (see Remark 3.6). Moreover, the holonomy $h_{x}$ is equal to the holonomy $h(\gamma)$ from Remark 3.6. Similarly, $h_{y}=h(\psi(\gamma))$.

Since $\tilde{\psi}$ is an automorphism of $E$ as of a $\rho_{1}^{\star} \mathbb{A}_{1}$-representation, $\tilde{\psi}$ commutes with holonomy along $\rho_{1}^{\star} \mathbb{A}_{1}$-paths. The anti-isomorphism $J$ defined by (25) interchanges (up to a sign) the structures of $\rho_{1}^{\star} \mathbb{A}_{1^{-}}$and $\rho_{2}^{\star} \mathbb{A}_{2}$ representations on $E$. Therefore, $\tilde{\psi}$ also commutes with holonomy along $\rho_{2}^{\star} \mathbb{A}_{2}$-paths. In particular, $h(\psi(\gamma)) \tilde{\psi}=\tilde{\psi} h(\gamma)$ which completes the proof.

Remark 5.8 (Morita invariance of the modular class). One can show that the canonical line bundle (see Example 3.9) of $P_{1}$ goes to the canonical line bundle of $P_{2}$ under the correspondence (21); see [Cr1]. Passing to the first characteristic class turns the correspondence (21) into the isomorphism $I_{W}$. This shows that $I_{W}$ sends the modular class of $P_{1}$ to the modular class of $P_{2},[\mathrm{Cr} 1]$ :

$$
I_{W}\left(\bmod \left(P_{1}\right)\right)=\bmod \left(P_{2}\right)
$$

in the self-explanatory notation of [GG].

Let us outline a direct proof (26). Let $\mu_{1}$ and $\mu_{2}$ be volume forms on $P_{1}$ and $P_{2}$, respectively. Denote by $D$ the symplectic *-operator on $W$, [Br]. The forms $D \rho_{1}^{*} \mu_{1}$ and $\rho_{2}^{*} \mu_{2}$ have the same degree $\operatorname{dim} P_{2}$ and the same null-space $\operatorname{ker}\left(\rho_{2}\right)_{*}$. Hence,

$$
D \rho_{1}^{*} \mu_{1}= \pm e^{H} \rho_{2}^{*} \mu_{2}
$$

for some smooth function $H$ on $W$. One can easily verify that $\left(\rho_{1}\right)_{*} X_{H}=v_{1}$ and $\left(\rho_{2}\right)_{*} X_{H}=-v_{2}$, where $X_{H}$ is the Hamiltonian vector field of $H$ on $W$ 
and $v_{1}$ and $v_{2}$ are modular vector fields corresponding to $\mu_{1}$ and, respectively, $\mu_{2}$ (see [We2]). By Lemma 5.7, $I_{W}\left(\left[v_{1}\right]\right)=\left[v_{2}\right]$ and (26) follows.

Although explicit examples are still lacking, it is unlikely that the Fernandes characteristic classes of degree greater than two are in any sense invariant of Morita equivalence. This is one of the motivations for introducing weak Morita $l$-equivalence; see Section 6.2.

Proposition 5.9. Let $P$ be an integrable Poisson manifold and $F$ a simply connected symplectic manifold. Equip $P \times F$ with the product Poisson structure. Then $\operatorname{Vect}_{\pi}^{\text {hom }}(P)=\operatorname{Vect}_{\pi}^{\text {hom }}(P \times F)$ and $K U_{\pi}^{\text {hom }}(P)=K U_{\pi}^{\text {hom }}(P \times F)$.

Proof. Since $P$ is integrable, $P$ is Morita equivalent to $P \times F,[\mathrm{Xu} 1]$.

Of course, the assertion of Proposition 5.9 holds also for $K_{\pi}^{\text {def }}$. Moreover, in this case the integrability assumption can be omitted; see Proposition 6.5 below.

\section{Weak Morita Category.}

\subsection{Weak Morita equivalence.}

To extend the results of Section 5.2 to algebroids we adopt the following definition, which is a result of "axiomatization" of the proof of (21).

Definition 6.1. Let $P_{1}$ and $P_{2}$ be smooth manifolds equipped with algebroids $\mathbb{A}_{1}$ and $\mathbb{A}_{2}$, respectively. We say that $\left(P_{1}, \mathbb{A}_{1}\right)$ and $\left(P_{2}, \mathbb{A}_{2}\right)$ are weakly Morita equivalent if there exists a manifold $Q$, submersions

$$
P_{1} \stackrel{\rho_{1}}{\leftarrow} Q \stackrel{\rho_{2}}{\rightarrow} P_{2}
$$

with simply connected fibers, and an isomorphism between the pull-back algebroids $\rho_{1}^{\star} \mathbb{A}_{1}$ and $\rho_{2}^{\star} \mathbb{A}_{2}$.

It is clear that for Poisson manifolds weak Morita equivalence is indeed weaker than genuine Morita equivalence. Weak Morita equivalence has some properties similar to (M1)-(M3). For example, by Lemma 5.6, weakly Morita equivalent algebroids $\left(P_{1}, \mathbb{A}_{2}\right)$ and $\left(P_{2}, \mathbb{A}_{2}\right)$ have isomorphic the zeroth and first algebroid cohomology spaces. The algebroids $\rho_{1}^{\star} \mathbb{A}_{1}$ and $\rho_{2}^{\star} \mathbb{A}_{2}$ have the same foliation. Hence, $F \mapsto \rho_{2}\left(\rho_{1}^{-1}(F)\right)$, where $F$ runs through the leaves of $\mathbb{A}_{1}$, is a bijection between the leaves of $\mathbb{A}_{1}$ and $\mathbb{A}_{2}$. Moreover, 
the corresponding leaves have isomorphic first cohomology groups and even isomorphic fundamental groups as we will soon show. Finally, if $x_{1} \in P_{1}$ and $x_{2} \in P_{2}$ are such that $x_{1}=\rho_{1}(z)$ and $x_{2}=\rho_{2}(z)$ for some $z \in Q$, then $\mathbb{A}_{1}$ at $x_{1}$ and $\mathbb{A}_{2}$ at $x_{2}$ have isomorphic stabilizers.

The following result is a trivial consequence of Lemma 5.6.

Theorem 6.2. Assume that $\left(P_{1}, \mathbb{A}_{1}\right)$ and $\left(P_{2}, \mathbb{A}_{2}\right)$ are weakly Morita equivalent. Then $\operatorname{Vect}_{\mathbb{A}_{1}}\left(P_{1}\right) \cong \operatorname{Vect}_{\mathbb{A}_{2}}\left(P_{2}\right)$ and $\operatorname{Vect}_{\mathbb{A}_{1}}^{\text {def }}\left(P_{1}\right) \cong \operatorname{Vect}_{\mathbb{A}_{2}}^{\text {def }}\left(P_{2}\right)$.

Corollary 6.3. $K_{\mathbb{A}_{1}}^{\text {def }}\left(P_{1}\right) \cong K_{\mathbb{A}_{2}}^{\text {def }}\left(P_{2}\right)$.

Remark 6.4 (The category of representations). In the algebraic setting, Morita equivalent rings have equivalent categories of modules. The same is true for $C^{*}$-algebras, $[\mathrm{Ri}]$. For a fixed algebroid $\mathbb{A}$ over $P$, let $\mathcal{V}(\mathbb{A})$ be the category of representations of $\mathbb{A}$. It is easy to see that for a submersion $\rho: Q \rightarrow P$ with simply connected fibers, the pull-back $E \mapsto \rho^{*} E$ is an equivalence of categories $\mathcal{V}(\mathbb{A}) \cong \mathcal{V}\left(\rho^{\star} A\right)$. Hence, weakly Morita equivalent algebroids have equivalent categories of representations. It would be interesting to check whether the converse is true or not.

A dual approach to "modules" over Poisson manifolds is developed by Ping $\mathrm{Xu},[\mathrm{Xu} 1, \mathrm{Xu2}$ ], in the context of Morita equivalence of groupoids. In particular, $\mathrm{Xu}$ showed that integrable Poisson manifolds which are Morita equivalent have equivalent "categories" of symplectic realizations and "symplectic left modules", [Xu1, Xu2].

Proposition 6.5. Let $P$ be a Poisson manifold and $F$ a simply connected symplectic manifold. Equip $P \times F$ with the product Poisson structure. Then $P$ and $P \times F$ are weakly Morita equivalent. Thus $\operatorname{Vect}_{\pi}(P) \cong \operatorname{Vect}_{\pi}(P \times F)$ and $\operatorname{Vect}_{\pi}^{\operatorname{def}}(P) \cong \operatorname{Vect}_{\pi}^{\text {def }}(P \times F)$. As a consequence, $K_{\pi}^{\text {def }}(P)=K_{\pi}^{\text {def }}(P \times$ $F)$.

Proof. Let $\rho$ be the natural projection $P \times F \rightarrow P$. It suffices to show that $\rho^{\star} T^{*} P$ is isomorphic to $T^{*}(P \times F)$. In the notation of the proof of Theorem 5.3 , the isomorphism is $(\alpha, X) \mapsto \alpha+\iota_{X_{F}} \omega$. Here $X_{F}$ is the $F$-component of $X$, the form $\omega$ is the symplectic form on $F$, and $\iota_{X_{F}} \omega$ is extended to a genuine differential form on $P \times F$ using the direct product structure.

Although weak Morita equivalence does not enable one to handle homogeneous vector bundles, it is in some sense a "better behaving" notion than Morita equivalence of Poisson manifolds: 
Proposition 6.6. Weak Morita equivalence is an equivalence relation.

We will prove this proposition in Section 6.2.

Let us now consider some examples of weak Morita equivalence. The first two of these are simply minor variations on the theme of the Morita equivalence classification of symplectic manifolds by their fundamental groups; see [Xu1, Proposition 2.1].

Example 6.7. Let $\mathbb{A}_{1}=T P_{1}$ and $\mathbb{A}_{2}=T P_{2}$. Assume that $P_{1}$ and $P_{2}$ are connected. The pairs $\left(P_{1}, T P_{1}\right)$ and $\left(P_{2}, T P_{2}\right)$ are weakly Morita equivalent if and only if $\pi_{1}\left(P_{1}\right)$ and $\pi_{1}\left(P_{2}\right)$ are isomorphic (cf. [Xu1, Proposition 2.1]).

Indeed, as follows from Proposition 7.1, weakly Morita equivalent manifolds always have isomorphic fundamental groups. To prove the converse, set $Q=\left(\widetilde{P}_{1} \times \widetilde{P}_{2}\right) / \Gamma$. Here $\widetilde{P}_{1}$ and $\widetilde{P}_{2}$ are universal coverings of $P_{1}$ and $P_{2}$, respectively, and $\Gamma$ is the graph in $\pi_{1}\left(P_{1}\right) \times \pi_{1}\left(P_{2}\right)$ of an isomorphism $\pi_{1}\left(P_{1}\right) \cong \pi_{1}\left(P_{2}\right)$. Clearly, the natural projections $\rho_{1}: Q \rightarrow P_{1}$ and $\rho_{2}: Q \rightarrow P_{2}$ have simply connected fibers. Furthermore, by Example 5.5, we have isomorphisms $\rho_{1}^{\star} T P_{1} \cong T Q \cong \rho_{2}^{\star} T P_{2}$.

Example 6.8. Let $P_{1}$ and $P_{2}$ be connected symplectic manifolds with algebroids $T^{*} P_{1}$ and $T^{*} P_{2}$, respectively. The pairs $\left(P_{1}, T^{*} P_{1}\right)$ and $\left(P_{2}, T^{*} P_{2}\right)$ are weakly Morita equivalent if and only if $\pi_{1}\left(P_{1}\right)$ and $\pi_{1}\left(P_{2}\right)$ are isomorphic. By [Xu1, Proposition 2.1], this is equivalent to that $P_{1}$ and $P_{2}$ are Morita equivalent. Indeed, for weak Morita equivalence, algebroids matter only up to isomorphism. On symplectic manifolds anchor maps are isomorphisms of algebroids: $T^{*} P_{1} \cong T P_{1}$ and $T^{*} P_{2} \cong T P_{2}$. The assertion follows now from Example 6.7.

Example 6.9. Let $P_{1}$ and $P_{2}$ be equipped with zero Poisson structures. (Hence, the algebroids are again $T^{*} P_{1}$ and $T^{*} P_{2}$, but both anchor maps are now zero.) Then $P_{1}$ and $P_{2}$ are weakly Morita equivalent if and only if these manifolds are diffeomorphic. Indeed, in this case the submersions of a weak Morita equivalence $P_{1} \stackrel{\rho_{1}}{\leftarrow} Q \stackrel{\rho_{2}}{\rightarrow} P_{2}$ must have the same fibers.

Example 6.10. Let $P_{1}$ and $P_{2}$ carry foliations $\mathcal{F}_{1}$ and $\mathcal{F}_{2}$ and let $\mathbb{A}_{1}=T \mathcal{F}_{1}$ and $\mathbb{A}_{2}=T \mathcal{F}_{2}$. Then $\left(P_{1}, \mathbb{A}_{1}\right)$ and $\left(P_{2}, \mathbb{A}_{2}\right)$ are weakly Morita equivalent if and only if there exist submersions $P_{1} \stackrel{\rho_{1}}{\leftarrow} Q \stackrel{\rho_{2}}{\rightarrow} P_{2}$ with simply connected fibers and such that the foliations $\rho_{1}^{*} \mathcal{F}_{1}$ and $\rho_{2}^{*} \mathcal{F}_{2}$ coincide.

Remark 6.11. Weak Morita equivalence of algebroids defined above is an infinitesimal version equivalence of topological groupoids [MRW]. It would 
be interesting to check if weak Morita equivalence of algebroids is equivalent to Morita equivalence of groupoids in the integrable case. ${ }^{5}$ For instance, in the above examples all algebroids are integrable and Morita equivalence for the corresponding groupoids is obvious.

\subsection{Weak Morita category.}

Already the argument in [GL] suggested that Morita equivalence is an isomorphism in some category. In this section, we introduce the corresponding category using weak Morita equivalence which affords more flexibility than genuine Morita equivalence.

Fix a pair of integers $(k, l)$ with $0 \leq k \leq l$. A weak Morita $(k, l)$ morphism from an algebroid $\left(P_{1}, \mathbb{A}_{1}\right)$ to an algebroid $\left(P_{2}, \mathbb{A}_{2}\right)$ is a pair of submersions $P_{1} \stackrel{\rho_{1}}{\leftarrow} Q \stackrel{\rho_{2}}{\rightarrow} P_{2}$ such that $\rho_{1}$-fibers are $l$-connected and $\rho_{2}$-fibers are $k$-connected and an isomorphism of algebroids $\rho_{1}^{\star} \mathbb{A}_{1} \cong \rho_{2}^{\star} \mathbb{A}_{2}$. (See $[\mathrm{HM}]$ for the definition of an isomorphism of algebroids.)

In what follows, we identify two morphisms between the same pairs when there exists an diffeomorphism between their middle terms, $Q$ and $Q^{\prime}$, and isomorphisms between the pull-back algebroids over $Q$ and $Q^{\prime}$ such that all the diagrams that arise are commutative.

Let us define the composition of two weak Morita $(k, l)$-morphisms

$$
\left(P_{1}, \mathbb{A}_{1}\right) \stackrel{\rho_{1}}{\leftarrow} Q \stackrel{\sigma_{1}}{\rightarrow}(P, \mathbb{A}) \quad \text { and } \quad(P, \mathbb{A}) \stackrel{\sigma_{2}}{\leftarrow} S \stackrel{\rho_{2}}{\rightarrow}\left(P_{2}, \mathbb{A}_{2}\right) .
$$

Let $W \subset Q \times S$ be the preimage of the diagonal under $\left(\sigma_{1}, \sigma_{2}\right): Q \times S \rightarrow$ $P \times P$. It follows by transversality that $W$ is a smooth submanifold of $Q \times S$. For $(x, y) \in W$ we set $\lambda_{1}(x, y)=\rho_{1}(x)$ and $\lambda_{2}(x, y)=\rho_{2}(y)$. It is easy to see that the composition is well-defined. (Recall that a morphism is really an equivalence class.)

Theorem 6.12. $\left(P_{1}, \mathbb{A}_{1}\right) \stackrel{\lambda_{1}}{\leftarrow} W \stackrel{\lambda_{2}}{\rightarrow}\left(P_{2}, \mathbb{A}_{2}\right)$ is a weak Morita $(k, l)$ morphism with the isomorphism $\lambda_{1}^{\star} \mathbb{A}_{1} \cong \lambda_{2}^{\star} \mathbb{A}_{2}$ given by (27) below.

A routine verification, which we omit, now implies

Corollary 6.13. The weak Morita $(k, l)$-category is indeed a category.

Note that the identity map in this category is $P \stackrel{i d}{\leftarrow} P \stackrel{i d}{\rightarrow} P$. We call the pairs $\left(P_{1}, \mathbb{A}_{1}\right)$ and $\left(P_{2}, \mathbb{A}_{2}\right)$ weakly Morita l-equivalent when there is a

\footnotetext{
${ }^{5}$ The author is grateful to Rui Loja Fernandes for this remark.
} 
weak Morita $(l, l)$-morphism between these pairs (but not necessarily an isomorphism). By symmetry, weak Morita $l$-equivalence is an equivalence relation.

Proof of Proposition 6.6. Weak Morita equivalence is just morphism in the weak Morita (1,1)-category.

Proof of Theorem 6.12. We focus on the properties of $\lambda_{1}$. For $\lambda_{2}$ the reasoning is identical.

To show that $\lambda_{1}$ is a submersion, we need to verify that this map is onto and so is its derivative $\left(\lambda_{1}\right)_{*}$. The surjectivity is a pure set-theoretic fact. (Let $z \in P_{1}$. Take any $x \in \rho_{1}^{-1}(z)$ and any $y \in S$ with $\sigma_{1}(x)=\sigma_{2}(y)$. Then $\lambda_{1}(x, y)=z$.) This argument uses only the assumption that all maps involved are onto. Hence, the surjectivity also holds in the category of linear spaces and, in particular, $\left(\lambda_{1}\right)_{*}$ is onto.

A similar argument shows that the natural projections $Q \stackrel{p_{1}}{\leftarrow} W \stackrel{p_{2}}{\rightarrow} S$ are also submersions.

Let us check that for $z \in P_{1}$, the fiber $\lambda_{1}^{-1}(z)$ is $l$-connected. The projection $\lambda_{1}^{-1}(z) \rightarrow \rho_{1}^{-1}(z)$ sending $(x, y)$ to $x$ is a submersion whose fiber over $x$ is $\sigma_{2}^{-1}\left(\sigma_{1}(x)\right)$. Hence, all fibers and the range of this submersion are $l$-connected. By Proposition 7.1, which we state and prove in the next section, $\lambda_{1}^{-1}(z)$ is $l$-connected.

It remains to define the isomorphism $\lambda_{1}^{\star} \mathbb{A}_{1} \cong \lambda_{2}^{\star} \mathbb{A}_{2}$. First note the factorizations

$$
\lambda_{1}=\rho_{1} p_{1}, \quad \lambda_{2}=\rho_{2} p_{2} \quad \text { and } \quad \sigma_{1} p_{1}=\sigma_{2} p_{2} .
$$

Using functoriality of the pull-back, $[\mathrm{HM}]$, we set this isomorphism to be

$$
\lambda_{1}^{\star} \mathbb{A}_{1}=p_{1}^{\star} \rho_{1}^{\star} \mathbb{A}_{1} \cong p_{1}^{\star} \sigma_{1}^{\star} \mathbb{A}=p_{2}^{\star} \sigma_{2}^{\star} \mathbb{A} \cong p_{2}^{\star} \rho_{2}^{\star} \mathbb{A}_{2}=\lambda_{2}^{\star} \mathbb{A}_{2},
$$

where the isomorphisms are induced by the isomorphisms of the algebroids from the original weak Morita morphisms. This concludes the proof.

By a straightforward inspection of the definition, we obtain

Proposition 6.14. The algebroid cohomology up to degree $l$ and, for $l \geq 1$, Vect, Vect ${ }^{\text {def }}$, and $K^{\text {def }}$ are contravariant functors on the weak Morita $(k, l)$ category. 
In particular, weak Morita $(k, l)$-morphisms induce isomorphisms of algebroid cohomology up to degree $k$ and also in Vect, Vect ${ }^{\text {def }}$, and $K^{\text {def }}$ when $k \geq 1$. Note also that the cohomology assertion in Proposition 6.14 generalizes a result obtained in [Cr1] in the integrable case.

Remark 6.15. With the present equivalence of morphisms, the weak Morita $(k, l)$-category has very few isomorphisms. A $(k, l)$-morphism is an isomorphism if and only if both $\rho_{1}$ and $\rho_{2}$ are diffeomorphisms. (This can be seen by a simple dimension count together with the description of $\lambda_{1}$ and $\lambda_{2}$ in the proof of Theorem 6.12.) For example, consider the $(l, l)$-category. The morphisms $P_{1} \stackrel{\rho_{1}}{\leftarrow} Q \stackrel{\rho_{2}}{\rightarrow} P_{2}$ and $P_{2} \stackrel{\rho_{2}}{\leftarrow} Q \stackrel{\rho_{1}}{\rightarrow} P_{1}$ are inverse to each other if and only if $\rho_{1}$ and $\rho_{2}$ are diffeomorphisms. This somewhat counterintuitive property suggests that, perhaps, one should weaken the equivalence relation defining morphisms.

Some examples of weak Morita $(k, l)$-morphisms can be readily found by modifying Examples 6.7-6.10. The next three examples farther illustrate the naturality of various constructions under weak Morita morphisms.

Example 6.16 (Weak Morita invariance of characteristic classes). Consider the homomorphism of algebroid cohomology $H^{*}\left(\mathbb{A}_{2}\right) \rightarrow H^{*}\left(\mathbb{A}_{1}\right)$ induced by a weak Morita $(k, l)$-morphism from $\left(P_{1}, \mathbb{A}_{1}\right)$ to $\left(P_{2}, \mathbb{A}_{2}\right)$. Then the Fernandes characteristic classes, [Fe2], of $\mathbb{A}_{2}$ up to degree $l$ are mapped to the Fernandes characteristic classes of $\mathbb{A}_{1}$. This follows from the fact that the pull-back $H^{*}(\mathbb{A}) \rightarrow H^{*}\left(\rho^{\star} \mathbb{A}\right)$, for a submersion $\rho$, sends the characteristic classes of $\mathbb{A}$ to those of $\rho^{\star} \mathbb{A}$.

Additionally, as is easy to see, the canonical line bundle of $\mathbb{A}_{1}$ corresponds to the canonical line bundle of $\mathbb{A}_{2}$ (see Remarks 3.9 and 5.8).

Example 6.17 (Weak Morita invariance of Poisson homology). Let $P_{1}$ and $P_{2}$ be two weakly Morita $l$-equivalent Poisson manifolds. Then the Poisson homology of $P_{1}$ and $P_{2}$ are isomorphic up to co-degree l, i.e., $H_{n_{1}-j}^{\pi}\left(P_{1}\right)=H_{n_{2}-j}^{\pi}\left(P_{2}\right)$ for $j \leq l$, where $n_{1}=\operatorname{dim} P_{1}$ and $n_{2}=\operatorname{dim} P_{2}$. (See [Br] for the definition of Poisson homology; see also [GG] for a much weaker version of this result.) This follows from the duality between the Poisson homology and the cohomology twisted by the canonical line bundle, [ELW], and from the fact that the canonical line bundles of $P_{1}$ and $P_{2}$ are mapped to each other by the equivalence. (The reader should be aware that the duality is described in [ELW] in different terms.) This example also generalizes to algebroids. 
Example 6.18. A weak Morita $l$-equivalence induces isomorphisms of homotopy and (co)homology groups up to degree $l$. This is a consequence of Proposition 7.1.

Remark 6.19. The definition of weak Morita $(k, l)$-category is only one out of a few inequivalent, but similar, constructions with the same properties. For instance, one may require the fibers to be simply connected and have vanishing real cohomology up to degree $k$ or $l$. All of the above results would still hold for this alternative definition. Another possible modification is to replace the isomorphism $\rho_{1}^{\star} \mathbb{A}_{1} \cong \rho_{2}^{\star} \mathbb{A}_{2}$ by a morphisms of algebroids $\rho_{1}^{\star} \mathbb{A}_{1} \rightarrow \rho_{2}^{\star} \mathbb{A}_{2}$. (See also Remark 6.15.)

This account of the weak Morita category is by no means intended to be complete or detailed. Its only goal is to show that the concept is natural and of some use and many questions are deliberately left out. For instance: let $\mathcal{F}_{1}$ on $P_{1}$ and $\mathcal{F}_{2}$ on $P_{2}$ be Morita $l$-equivalent foliations. Does the isomorphism from Example 6.18 preserve their characteristic classes (in the de Rham cohomology) up to degree $l$ ?

\section{Appendix: Submersions with l-connected fibers.}

The goal of this appendix is to prove the following

Proposition 7.1. Let $\rho: X \rightarrow Y$ be a submersion whose fibers are $l$ connected. Then $\rho$ induces an isomorphism of homotopy groups $\pi_{n}(X) \cong$ $\pi_{n}(Y)$ for $n \leq l$.

Remark 7.2. When $\rho$ is a fibration, e.g., the fibers of $\rho$ are compact, Proposition 7.1 follows immediately from the long exact sequence of homotopy groups.

A statement more general than Proposition 7.1 was announced in [Me]. Below, for the sake of completeness, we prove the proposition.

Proof of Proposition 7.1. For simplicity, let us focus on the case where $Y$ is $l$-connected. Then we need to show that $X$ is also $l$-connected. The proof of the general case requires only superficial modifications.

Let us first set notations and terminology. In what follows, $B$ and $K$ will be compact manifolds possibly with boundary or even with corners. (In fact, these will be either the sphere $S^{n}$, the $n$-ball $D^{n}$, or the $n$-cube $I^{n}$.) 
For maps $f: B \rightarrow Y$ and $F: B \times K \rightarrow X$, we say that $F$ covers $f$ or that $F$ is a lift of $f$ if $\rho(F(b, y))=f(b)$ for all $b \in B$ and $y \in K$, i.e., the diagram

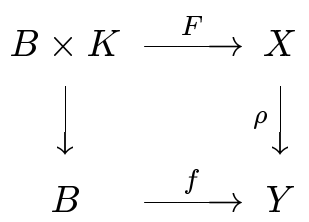

is commutative. The same applies to homotopies $f_{t}$ and $F_{t}$, where $t$ is in some interval.

Let us now establish some preliminary facts needed for the proof.

Lemma 7.3 (Local extensions of lifts). Let $f_{t}: B \rightarrow Y, t \in(-1,1)$, be a continuous homotopy and $F_{0}: B \times K \rightarrow X$ a continuous lift of $f_{0}$. Then for a small $\epsilon>0$, there exists a continuous lift $F_{t}$ of $f_{t}$ for $t \in(-\epsilon, \epsilon)$.

The lemma is clear: Since $F_{0}(B \times K)$ is compact, this set has a neighborhood $U$ such that the restriction of $\rho$ to $U$ is a trivial fiber bundle.

Lemma 7.4 (Matching homotopies). Let $f_{t}: B \rightarrow Y, t \in[-1,1]$, be $a$ smooth homotopy. Assume that there is

(i) a continuous lift $F_{t}^{+}: B \times K \rightarrow X$ of $f_{t}$ for $t \in[-1,0]$,

(ii) a continuous lift $F_{t}^{-}: B \times K \rightarrow X$ of $f_{t}$ for $t \in[0,1]$ such that these two lifts coincide on $B \times \partial K$ for $t=0$, i.e., $F_{0}^{-}(B \times$ $\partial K)=F_{0}^{+}(B \times \partial K)$, and

(iii) a continuous homotopy $G_{s}, s \in[0,1]$, relative $B \times \partial K$ between $F_{0}^{-}$and $F_{0}^{+}$covering $f_{0}$. (We call $G$ a connecting homotopy.)

Then there exists a continuous lift $F_{t}, t \in[-1,1]$, of $f_{t}$ which is equal to $F_{t}^{ \pm}$ for $t$ near \pm 1 .

We emphasize that this lemma is nearly obvious. In fact, by inserting $G$ between $F^{-}$and $F^{+}$, we obtain a homotopy which is almost a lift of $f$. This homotopy requires only a re-parameterization and a minor alteration in the middle part to be turned into a genuine lift of $f$. For the sake of completeness we give a detailed proof.

Proof. Assume first that $Y=B \times[-1,1]$ and the homotopy $f$ is just the identity map. Let

$$
Z=\bigcup_{s \in[0,1]} G_{s}(B \times K) \subset \rho^{-1}(B \times 0) .
$$


This is a compact set. It is easy to see that there exists a neighborhood $U$ of $Z$ in $\rho^{-1}(B \times 0)$, a small $\epsilon>0$, and an embedding $U \times(-\epsilon, \epsilon) \hookrightarrow X$ such that $\rho(x, t)=(\rho(x), t)$, where $x \in U$ and $t \in(-\epsilon, \epsilon)$. Here and in what follows, we identify $U \times(-\epsilon, \epsilon)$ with a neighborhood of $Z$ in $X$. For small $|t|$, the homotopies $F_{t}^{ \pm}$take values in this neighborhood.

It is also easy to see that $F_{t}^{ \pm}$can be changed for small $|t|$ so as to make $F_{t}^{ \pm}$independent of $t$ in $U \times(-\epsilon, \epsilon)$. (In other words, the $U$-component of $F_{t}^{ \pm}(y)$ is constant for all $y \in B \times K$ and $|t|<\delta<\epsilon$.) From now on we assume that $F_{t}^{ \pm}$have this property. (The resulting maps can be chosen of the same smoothness class as the original ones.)

Let $\phi:[-\delta, \delta] \rightarrow[0,1]$ be a smooth monotone increasing function which is identically equal to 0 for $t$ near $-\delta$ and to 1 for $t$ near $\delta$. The required lift is then given by the formula

$$
F_{t}= \begin{cases}F_{t}^{-} & \text {for } t \in[-1,-\delta], \\ G_{\phi(t)} & \text { for } t \in[-\delta, \delta], \\ F_{t}^{+} & \text {for } t \in[\delta, 1] .\end{cases}
$$

Let us now prove the lemma for a general smooth homotopy $f: B \times$ $[-1,1] \rightarrow X$. Consider the pull-back submersion $f^{*} \rho: \tilde{Y} \rightarrow B \times[-1,1]$ with the domain

$$
\tilde{Y}=\{(a, y) \in(B \times[-1,1]) \times Y \mid f(a)=\rho(y)\} .
$$

By transversality, $\tilde{Y}$ is smooth and $f^{*} \rho:(a, y) \mapsto a$ is a submersion. A lift of $f$ pulls back to a continuous lift of $i d$. (In fact, this pull-back is of the same smoothness class as the original lift, as one can check in local coordinates in the domain of $f$ ). Thus $F^{+}, F^{-}$, and $G$ pull back to maps satisfying the hypotheses of the lemma and covering $i d$. For these pull-backs the existence of the lift $F$ has already been proved. To obtain a lift of $f$, it remains to compose this lift with the natural map $\tilde{Y} \rightarrow Y$.

The key step in the proof is the following

Lemma 7.5 (Fillings of lifts). Let $B$ be the closed ball $D^{n}$ or the cube $I^{n}$ or the sphere $S^{n}$ and $K=S^{m}$. Let $F: B \times K \rightarrow X$ be a continuous lift of a smooth map $f: B \rightarrow Y$. Then, for $n+m \leq l$, there exists a continuous lift $\Phi: B \times D^{m+1} \rightarrow X$ of $f$ (called a filling of $F$ ) whose restriction to $B \times K=B \times \partial D^{m+1}$ is $F$.

We will need this lemma only for $m=0$, but it is more convenient to prove it for all $m$ with $n+m \leq l$. 
Proof of Lemma 7.5 for $B=D^{n}$ and $I^{n}$. We will prove the lemma for $B=I^{n}$. (This implies the lemma for $B=D^{n}$, for $f$ can always be extended from $D^{n}$ to some $n$-cube containing $D^{n}$ and $F$ can also be extended to $I^{n} \times K$.) The proof is by induction in $n$.

For $n=0$, i.e., $I^{0}=\{p t\}$, the assertion follows from the assumptions that the fibers of $\rho$ are $l$-connected and $m \leq l$. Assume that the assertion is proved for $\operatorname{dim} B<n$.

Set $I^{n}=I^{n-1} \times[0,1]$ and $C_{t}=I^{n-1} \times\{t\}$ for $t \in[0,1]$. Let also $f_{t}=\left.f\right|_{C_{t}}$ and $F_{t}=\left.F\right|_{C_{t} \times K}$. By the induction hypothesis, $F_{0}$ has a filling $\bar{\Phi}_{0}$ and hence, by Lemma 7.3, this filling extends over $I^{n-1} \times[0, \epsilon)$ for some small $\epsilon>0$. Consider the maximal open in $[0,1]$ interval $[0, r)($ or $[0,1])$ over which a filling $\bar{\Phi}_{t}$ of $F_{t}$ exists. If this interval is $[0,1]$ the proof is finished. Hence, let us assume that the interval is $[0, r)$ with $0<r \leq 1$ and arrive to a contradiction. We will present the proof for $r<1$. The argument for $r=1$ requires only minor modifications.

By the induction hypothesis, $F_{r}: C_{r} \times K \rightarrow X$ admits a filling, which extends to a filling $\widetilde{\Phi}_{t}$ over some small interval $[r-\epsilon, r+\epsilon]$, by Lemma 7.3. Now we have two fillings, $\bar{\Phi}_{r-\epsilon}$ and $\widetilde{\Phi}_{r-\epsilon}$, of $F_{r-\epsilon}$. These are maps $C_{r-\epsilon} \times D^{m+1} \rightarrow X$ which coincide on $C_{r-\epsilon} \times S^{m}$ and hence give rise to a map $C_{r-\epsilon} \times S^{m+1} \rightarrow X$ covering $f_{r-\epsilon} \cdot{ }^{6}$

The induction hypothesis applies to this map, for $C_{r-\epsilon}$ is a cube of dimension $n-1$ and $(n-1)+(m+1)=n+m \leq l$. Thus it has a filling, which can be thought of as a homotopy $G$ between $\bar{\Phi}_{r-\epsilon}$ and $\widetilde{\Phi}_{r-\epsilon}$ which covers $f_{r-\epsilon}$.

Lemma 7.4 applies to the homotopy $\bar{\Phi}_{t}$ for $t \in[0, r-\epsilon]$, the homotopy $\widetilde{\Phi}_{t}$ for $t \in[r-\epsilon, r+\epsilon]$, and the connecting homotopy $G$. As a result, we obtain a filling of $F_{t}$ for $t \in[0, r+\epsilon]$. This contradicts the choice of $r$.

Proof of Lemma 7.5 for $B=S^{n}$. As in the proof for $B=D^{n}$, we argue inductively. For $n=0$ and $m \leq l$, the image $F\left(S^{0} \times S^{m}\right)$ is comprised of two mapped $m$-spheres contained in the fibers of $\rho$. Since these fibers are $l$-connected, the filling does exist.

Assuming that the lemma is proved for $\operatorname{dim} B<n$, we establish it for $S^{n}$. Let us think of $S^{n}$ as the union of two hemispheres: $S^{n}=D_{-}^{n} \cup D_{+}^{n}$.

\footnotetext{
${ }^{6}$ This map may fail to be smooth at the points of $C_{r-\epsilon} \times S^{m}$ even if all the maps considered prior to this moment are. Although a smoothing argument can be applied here, we prefer to work with continuous lifts. In fact, this is the only reason why we ever need to drop the smoothness assumption; the proofs of Lemmas 7.3 and 7.4 go through in the smooth category.
} 
Lemma 7.5 has already been proved for the discs and, hence, it applies to $F_{ \pm}=\left.F\right|_{D_{ \pm}^{n} \times S^{m}}$. As a result we obtain two fillings $\Phi_{ \pm}$over the hemispheres. Along the "equator" $S^{n-1} \times S^{m}$ of $S^{n} \times S^{m}, \Phi_{ \pm}$are fillings of the same map $\left.F\right|_{S^{n-1} \times S^{m}}$. Hence, $\Phi_{ \pm}$give rise to a continuous map $S^{n-1} \times S^{m+1} \rightarrow X$ covering $\left.f\right|_{S^{n-1}}$. By the induction hypothesis, this map has a filling and this filling can be viewed as a connecting homotopy $G$ between $\left.\Phi_{-}\right|_{S^{n-1} \times S^{m}}$ and $\left.\Phi_{+}\right|_{S^{n-1} \times S^{m}}$. Applying Lemma 7.4 to $\Phi_{-}, \Phi_{+}$, and $G$, we obtain the required filling for $F$.

Lemma 7.6 (Lifting discs). For every smooth map $f: D^{n} \rightarrow Y$, where $n \leq l+1$, there exists a continuous lift $F: D^{n} \rightarrow X$.

Remark 7.7. Once a continuous lift is found, it is not hard show that a smooth lift also exists.

Proof of Lemma 7.6. We only outline the argument, for it is very similar to the proof of Lemma 7.5. As in that proof we replace $D^{n}$ by $I^{n}$.

Arguing inductively, we assume that the lemma is proved for the cubes of dimension less than $n$. As above, we set $C_{t}=I^{n-1} \times\{t\}$ and $f_{t}=\left.f\right|_{C_{t}}$, where $I^{n}=I^{n-1} \times[0,1]$, and consider the maximal open interval in $[0,1]$ over which the lift exists and which contains 0 . Denote this lift by $\bar{F}_{t}$. Let us assume that the interval has the form $[0, r)$ with $0<r \leq 1$ and arrive to contradiction. As in the proof of Lemma 7.5, we focus on the case $r<1$. (The case $r=1$ is handled similarly.)

By the induction assumption, we can lift $f_{r}$ to $X$ and then, using Lemma 7.3 , extend it to a homotopy $\widetilde{F}_{t}, t \in[r-\epsilon, r+\epsilon]$, for a small $\epsilon>0$. Thus we have two lifts of $f_{r-\epsilon}$, namely, $\bar{F}_{r-\epsilon}$ and $\widetilde{F}_{r-\epsilon}$. Together these lifts give rise to a map $I^{n-1} \times S^{0}$ covering $f_{r-\epsilon}$. Applying Lemma 7.5 , we find a filling for this map. This filling is nothing else but a homotopy between $\bar{F}_{r-\epsilon}$ and $\widetilde{F}_{r-\epsilon}$ covering $f_{r-\epsilon}$. By Lemma 7.4, the lift of $f_{t}$ extends to the interval $[0, r+\epsilon]$, which contradicts the choice of $r$.

Now we are in a position to finish the proof of Proposition 7.1 under the assumption that $Y$ is $l$-connected. Let $F_{0}: S^{n} \rightarrow X$, where $n \leq l$, be a smooth map and let $f: D^{n+1} \rightarrow Y$ be a smooth homotopy of $\rho F_{0}=\left.f\right|_{S^{n-1}}$ to a point. We will find a continuous homotopy $F: D^{n+1} \rightarrow X$ of $F_{0}$ to a point. By Lemma 7.6, there exists a lift $\bar{F}: D^{n+1} \rightarrow X$ of $f$. Furthermore, by Lemma 7.3, $F_{0}$ can be extended to a little closed collar $U$ of $S^{n}$ in $D^{n+1}$. Let $\widetilde{F}$ be this extension. Denote by $C$ the inner boundary of $U$ in $D^{n+1}$. 
Thus $C$ is the sphere $S^{n}$ over which we have two lifts $\left.\bar{F}\right|_{C}$ and $\left.\widetilde{F}\right|_{C}$ of $\left.f\right|_{C}$. These lifts comprise a map $C \times S^{0} \rightarrow X$ covering $\left.f\right|_{C}$, which, by Lemma 7.5, have a filling. This filling is a homotopy $G$ between $\left.\bar{F}\right|_{C}$ and $\left.\widetilde{F}\right|_{C}$ covering $\left.f\right|_{C}$. Applying Lemma 7.4, we obtain the required lift $F$.

In Proposition 7.1 (and in Lemma 7.6) the assumption that $\rho$ is a submersion is essential and cannot be replaced by the assumption that $\rho$ is onto.

Example 7.8. We start with an example where $Y$ and all fibers are connected, but $X$ is not. Let $X$ be the disjoint union of three copies of $\mathbb{R}$ which we denote by $X_{-}, X_{0}$, and $X_{+}$and let $Y=\mathbb{R}$. Let $f$ map diffeomorphically $X_{-}$and $X_{+}$onto $(-\infty, 0)$ and, respectively, $(0, \infty)$ and set $f\left(X_{0}\right)=0$. Every fiber is either a point or $X_{0}=\mathbb{R}$ and $Y$ is also connected. However, $X$ has three connected components.

Next we give an example where all fibers and $Y$ are simply connected, but $X$ is not simply connected. Consider a smooth map $\rho: \mathbb{R}^{2} \rightarrow \mathbb{R}$ such that $\rho^{-1}(0)$ is the strip $0 \leq x \leq 1$ in $\mathbb{R}^{2}$ and $\rho^{-1}(p)$ is a vertical line in $\mathbb{R}^{2}$ for every $p \neq 0$. (It is easy to see that such a map does exist.) Let now $X=\mathbb{R}^{2} \backslash\{(0,0)\}$ and $Y=\mathbb{R}$. Then a fiber of $\rho$ is either a vertical line or the strip $0 \leq x \leq 1$ with one point on its boundary deleted. Hence, all fibers of $\rho$ are simply connected and so is $Y$. However, $\pi_{1}(X)=\mathbb{Z}$.

\section{References.}

[Al] A.Yu. Alekseev, On Poisson actions of compact Lie groups on symplectic manifolds, J. Differential Geom., 45 (1997), 241-256.

[Be] M. Bertelson, Foliations associated to regular Poisson structures, Ph.D. Thesis, Stanford, 2000.

[Bo1] R. Bott, Lectures on characteristic classes of foliations, (Notes by Lawrence Conlon, with two appendices by J. Stasheff), Lectures on algebraic and differential topology (Second Latin American School in Math., Mexico City, 1971), Lecture Notes in Math., 279 (1972), 1-94. 
[Bo2] R. Bott, On some formulas for the characteristic classes of groupactions, in 'Differential topology, foliations and Gelfand-Fuks cohomology', Proc. Sympos., Pontifécia Univ. Catálica, Rio de Janeiro, 1976), Lecture Notes in Math., 652 (1978), 25-61.

[Br] J.-L. Brylinski, A differential complex for Poisson manifolds, J. Differential Geom., 28 (1988), 93-114.

[CdSW] A. Cannas da Silva and A. Weinstein, Lectures on geometric models for noncommutative algebras, Berkeley Mathematics Lecture Notes, 10, American Math. Soc., 1999.

[Co1] J. Conn, Normal forms for analytic Poisson structures, Ann. of Math., 119 (1984), 576-601.

[Co2] J. Conn, Normal forms for smooth Poisson structures, Ann. of Math., 121 (1985), 565-593.

[Co3] J. Conn, Correction to "Normal forms for analytic Poisson structures", Ann. of Math., 125 (1987), 433-436.

[Con1] A. Connes, A survey of foliations and operator algebras, in 'Operator Algebras and Applications', Proc. Symp. Pure Math, 38, ed. R.V. Kadison, Amer. math. Soc., 1982.

[Con2] A. Connes, Noncommutative Geometry, Academic Press, San Diego, 1994.

[Cr1] M. Crainic, Differentiable and algebroid cohomology, van Est isomorphisms, and characteristic classes, preprint 2000, math.DG/0008064.

[Cr2] M. Crainic, Connections up to homotopy and characteristic classes, preprint 2000, math.DG/0010085.

[ELW] S. Evens, J.-H. Lu and A. Weinstein, Transverse measures, the modular class, and a cohomology pairing for Lie algebroids, Quart. J. Math. Oxford Ser. (2), 50 (1999), 417-436.

[Fe1] R.L. Fernandes, Connections in Poisson geometry: Holonomy and invariants, preprint 2000, math. DG/0001129. 
[Fe2] R.L. Fernandes, Lie algebroids, holonomy and characteristic classes, preprint 2000, math. DG/0007132.

[Fu] D.B. Fuks, Cohomology of Infinite-Dimensional Lie Algebras, Consultants Bureau, NY, 1986.

[Gi] V.L. Ginzburg, Momentum mappings and Poisson cohomology, Internat. J. Math., 7 (1996), 329-358.

[GG] V.L. Ginzburg and A. Golubev, Holonomy on Poisson manifolds and the modular class, Israel J. Math., 122 (2001), 221-242, math.DG/9812153.

[GGK] V.L. Ginzburg, V. Guillemin and Y. Karshon, Moment Maps, Cobordisms, and Hamiltonian Group Actions, a book in preparation.

[GL] V.L. Ginzburg and J.-H. Lu, Poisson cohomology of Morita equivalent Poisson manifolds, IMRN, 10 (1992), 199-205.

[GW] V.L. Ginzburg and A. Weinstein, Lie-Poisson structures on some Poisson Lie groups, J. Amer. Math. Soc., 5 (1992), 445-453.

[He] G. Hector, Groupoids, feuilletages et $C^{*}$-algèbres (quelques aspects de la conjecture de Baum-Connes), Geometric study of foliations (Tokyo, 1993), World Sci. Publishing, River Edge, NJ, (1994), 334 .

[HM] P. Higgins and K. Mackenzie, Algebraic constructions in the category of Lie algebroids, J. Algebra, 129 (1990), 194-230.

[Hu] J. Huebschmann, Poisson cohomology and quantization, J. Reine Angew. Math., 408 (1990), 57-113.

[KT] F. Kamber and P. Tondeur, Foliated Bundles and Characteristic Classes, Lecture Notes in Math., 493 (1975).

[Lu] J.-H. Lu, Momentum mappings and reduction of Poisson actions, in 'Symplectic Geometry. Groupoids and Integrable Systems', Eds. P. Dazord and A. Weinstein, Séminaire Sud Rhodanien de Géométrie à Berkeley (1989), Math. Sci. Res. Inst. Publ., 20, Springer-Verlag, 1991. 
[LW] J.-H. Lu and A. Weinstein, Poisson Lie groups, dressing transformations, and Bruhat decompositions, J. Differential Geom., 31 (1990), 501-526.

[Ma] K. Mackenzie, Lie Groupoids and Lie Algebroids in Differential Geometry, Lecture Notes Series, 124, London Mathematical Society, 1987.

[Me] G. Meigniez, Sur le relévement des homotopies, C. R. Acad. Sci. Paris Sér. I Math., 321 (1995), 1497-1500.

[MM] I. Moerdijk and J. Mrčun, On integrability of infinitesimal actions, preprint 2000, math.DG/0006042.

[MRW] P. Muhly, J. Renault and D. Williams, Equivalence and isomorphism for groupoid $C^{*}$-algebras, J. Operator Theory, 17 (1987), $3-22$.

[Ol] R. Oliver, Vector bundles over classifying spaces, in 'Proceedings of the International Congress of Mathematicians', II, (Berlin, 1998), Doc. Math., 1998, extra Vol. II, 483-492.

[Ri] M.A. Rieffel, Induced representations of $C^{*}$-algebras, Advances in Math., 13 (1974), 176-257.

[Se] G. Segal, Classifying spaces and spectral sequences, Publ. Math. IHES, 34 (1968), 105-112.

[Va] I. Vaisman, On the geometric quantization of Poisson manifolds, J. Math. Phys., 32 (1991), 3339-3345.

[We1] A. Weinstein, The local structure of Poisson manifolds, J. Differential Geom., 18 (1983), 523-557.

[We2] A. Weinstein, The modular automorphism group of a Poisson manifold, J. Geom. Phys., 23 (1997), 379-394.

[We3] A. Weinstein, Poisson geometry. Symplectic geometry, Differential Geom. Appl., 9 (1998), 213-238.

[Xu1] P. Xu, Morita equivalence of Poisson manifolds, Comm. Math. Phys., 142 (1991), 493-509. 
[Xu2] P. Xu, Morita equivalent symplectic groupoids, in 'Symplectic geometry, groupoids, and integrable systems', Eds. P. Dazord and A. Weinstein, Séminaire Sud Rhodanien de Géométrie à Berkeley (1989), Springer-Verlag, Math. Sci. Res. Inst. Publ., 20, 1991, 291311.

Department of Mathematics

UC SANTA CRUZ

SANTA CruZ, CA 95064

E-mail address: ginzburg@math.ucsc.edu 